\title{
Robust design optimization in aeronautics using stochastic analysis and evolutionary algorithms
}

\author{
J Pons-Prats $^{1 *}$, G Bugeda ${ }^{2,3}$, F Zárate $^{3}$, and E Oñate ${ }^{3}$ \\ ${ }^{1}$ International Center for Numerical Methods in Engineering, Castelldefels, Spain \\ ${ }^{2}$ Universitat Politècnica de Catalunya, Barcelona, Spain \\ ${ }^{3}$ International Center for Numerical Methods in Engineering Barcelona, Spain
}

The manuscript was received on 14 September 2010 and was accepted after revision for publication on 11 May 2011.

DOI: $10.1177 / 0954410011412131$

\begin{abstract}
Uncertainties are a daily issue to deal with in aerospace engineering and applications. Robust optimization methods commonly use a random generation of the inputs and take advantage of multi-point criteria to look for robust solutions accounting with uncertainty definition. From the computational point of view, the application to coupled problems, like computational fluid dynamics (CFD) or fluid-structure interaction (FSI), can be extremely expensive. This study presents a coupling between stochastic analysis techniques and evolutionary optimization algorithms for the definition of a stochastic robust optimization procedure. At first, a stochastic procedure is proposed to be applied into optimization problems. The proposed method has been applied to both CFD and FSI problems for the reduction of drag and flutter, respectively.
\end{abstract}

Keywords: uncertainties, optimization, robust optimization, airfoil, aero-elastics

\section{INTRODUCTION}

Optimization problems are a meeting point of several disciplines. Thanks to the improvements in computer sciences, which enable fast computations, computational fluid dynamics (CFD) and aero-elastic problems have become a daily topic for analysis engineers. The trend on the design process is now focussed on dealing with uncertainties, which can greatly affect the final performance of the system.

Uncertainty is an important concept to be taken into consideration for simulation and optimization processes. Accounting with the management of uncertainties produces better results regarding the robustness of the design. Uncertainties related to

*Corresponding author: International Center for Numerical Methods in engineering (CIMNE), c/Esteve Terrades 5, 08860 Castelldefels, Spain.

email:jpons@cimne.upc.edu the quality of the analysis processes, like approximation of the modelling analytical equations, quality of the discretization, etc. can be taken into account, but here the focus will be on the uncertainties related with the definition of the real problem to be analysed. These uncertainties are usually related with the input variables for the analysis, which represents some natural behaviour, or some manufacturing parameters and tolerances. Uncertainties can be classified in two categories, as described by Helton and Davis [1]

The first one is the so-called random uncertainty: the behaviour of natural systems, with its inherent variability, is the best example. Thanks to empiric observation, random uncertainties can be accurately modelled and represented through the use of probabilistic methods.

The second category is the so-called epistemic uncertainty, which comes from a lack of knowledge of the system behaviour. Usually, it is not well represented nor modelled using classical probabilistic 
approaches and it leads to non-probabilistic methods based on interval specifications [2].

The aim of this study is to define a new methodology to be applied to optimization problems in aerodynamics, or coupled problems, enabling the stochastic definition of the input parameters for a better representation of uncertainties associated to input values. This methodology should be efficient, so it should reduce the number of needed calculations to a small amount compared with the dimensions of the search space. It should also be robust, and it should find the optimum value under a complex topology of the search space. It must be taken into consideration that engineering problems, and also those related to aerodynamics, or coupled problems, require a robust solution to ensure the optimal performance across the larger range of operation conditions as possible.

This article is organized in four sections. After this section, the second section focuses on the definition of the used stochastic procedure. The third section is devoted to the analysis of the integration of the stochastic procedure into optimization methods based

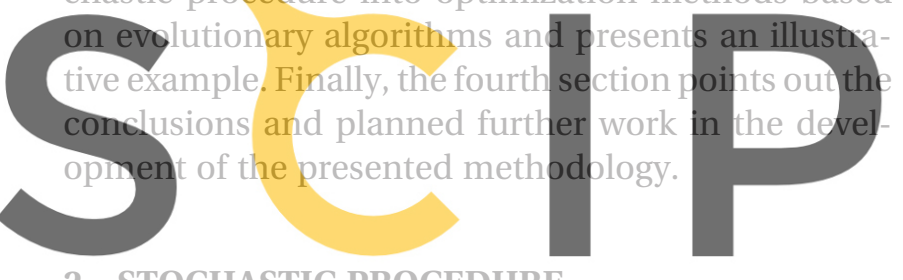

2 STOCHASTIC PROCEDURE

Register for free at https//www.scipedia.com tainties. From lack of information or knowledge of the analysed phenomena about intrinsic errors during tests, or numerical simulations, the parameters are always dealing with uncertainties. At the end, they can produce a big variability on the results. Not to take into account the possible variability of the different phenomena considered in the analysis can produce completely wrong conclusions. It is really important to ensure the best understanding of the phenomena, but also of the associated uncertainties and variability.

Stochastic procedures are based on the coupling between a generator of random values for those inputs with uncertainties and the analysis tool. Input variables with uncertainties, like flow boundary conditions, are defined using a probabilistic density function (PDF). Gaussian or uniform PDFs are the most commonly used. Then, a set of random values are generated for each random input variable following the corresponding PDF. Each of the randomly generated values represents a configuration of the problem to be analysed. The analyses of all the defined configurations produce a set of results that, at the end of the process, are analysed using statistical tools.

This section presents different examples of stochastic analysis taking into account the variability of different parameters of the problem, and the corresponding conclusions that can be extracted from the results.

Next tests are mainly intended to check if the stochastic procedure leads to meaningful results that can be used in further development of a stochastic robust design optimization method. Results will also be evaluated in comparison with real and known physical behaviour. It will provide a confirmation and validation of the whole procedure in two ways: regarding not only the procedure but also the meaning of the results.

In this study, the STAC code has been used for the generation of all random values. STAC is a stochastic analysis management tool. Thanks to the developments by Hurtado and Barbat [3] regarding the random generation of samples using Monte-Carlo and Latin Hypercube techniques, STAC tool provides a very friendly and easy to use user interface with pre-

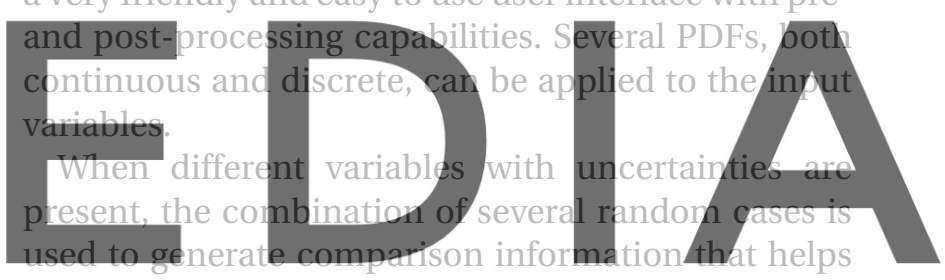

to identify the most relevant parameters regarding

o dowilityad the version without the watermark A CFD and aero-elastic stochastic analyses are shown in next sub-sections. The main aim of the CFD analysis is to analyse the variability of the lift and drag coefficients when angle of attack (AoA) and Mach number $(M)$ present uncertainties. The aero-elastic problem is defined to capture the variability of aerodynamic and structural values that can help to understand flutter phenomena, like lift $\left(C_{l}\right)$ and pressure drag $\left(C_{\mathrm{dp}}\right)$ coefficients, as well as angular spin and vertical deformations of the wing, when defining uncertainties for $M$, AoA, $x$-coordinate of the elastic axis position, the damping coefficients of wing, both angular and vertical movements, and the mass ratio. From an engineering point of view, the CFD problem can help to determine the best configuration regarding drag reduction and lift maximization, while the aero-elastic problem helps to analyse the flutter phenomena, which is of major importance due to safety reasons.

\subsection{Example of a stochastic CFD analysis}

In order to illustrate the main characteristics of a stochastic analysis, the analysis of a RAE2822 profile [4] 
using stochastically defined input parameters has been performed. The considered stochastic input parameters have been selected from the most relevant CFD values. Different probabilistic definitions have been applied to each of them in order to analyse the behaviour of the output data against the variability of the input data. The combination of those cases has enabled to detect the influence on the outputs of the modification of different input values.

Eight different stochastic analyses have been performed. Table 1 shows the characteristics of each of them. The first one has only considered the Mach number $(M)$ as a stochastic input variable, whereas the angle of attack (AoA) has been considered as fixed. On the other side, the second case has only considered the stochastic nature of AoA, whereas $M$ has been considered as fixed. Cases 3-8 have considered a stochastic nature for both the AoA and the $M$, but using different PDF definitions.

For all eight cases, STAC has defined a probabilistic set of values, 250 shots defined using Monte-Carlo method, according with the PDFs of each studied case. For each pair of input values, the corresponding

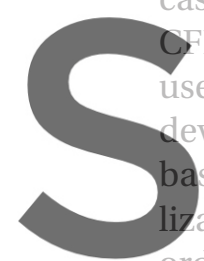

TD analysis has been pertormed. The CP code used in this study has been developed at CIMNE by lization technique added order to avoid non-physical solutions. An explicit multi-stage Runge-Kutta scheme is used as the time erformed. The CFD code
n PUMI, which is a code
lores and Ortega [5]. It is
quations and uses a stabi-
to the Galerkin scheme in
cal solutions. An explicit
cheme is used as the time Registeinfegrafreesaltehttps $b \mathrm{t}$ WWVW scipediancom tio solution. Special care has been taken in the code effi-
ciency in order to deal with complex geometrical problems avoiding high computational demands, i.e. minimum memory requirements, fast singletreaded performance, and a good parallel scaling. Other CFD tools could also be coupled into the procedure, like XFOIL [6], which deals with low subsonic problems, and which has been used for other tests not included in this article.

Outputs that have been obtained and statistically analysed are lift and pressure drag coefficients

Table 1 Definition of analysed cases

\begin{tabular}{lll}
\hline Flow conditions & $\begin{array}{l}\text { Angle of } \\
\text { attack Mean/SD }\end{array}$ & $\begin{array}{l}\text { Mach number } \\
\text { Mean/SD }\end{array}$ \\
\hline Case 1: Gaussian PDF & $4 /-$ & $0.7 / 0.08$ \\
Case 2: Gaussian PDF & $4 / 0.5$ & $0.7 /-$ \\
Case 3: Gaussian PDF & $4 / 0.5$ & $0.7 / 0.08$ \\
Case 4: Gaussian PDF & $4 / 1.0$ & $0.7 / 0.08$ \\
Case 5: Gaussian PDF & $4 / 1.5$ & $0.7 / 0.08$ \\
Case 6: Gaussian PDF & $4 / 0.5$ & $0.7 / 0.04$ \\
Case 7: Gaussian PDF & $4 / 0.5$ & $0.7 / 0.12$ \\
& Low-up bounds & Low-up bounds \\
\cline { 2 - 2 } Case 8: Uniform PDF & $2.5-4.5$ & $0.45-0.95$ \\
\hline
\end{tabular}

( $C_{l}$ and $C_{\mathrm{dp}}$ ). The solution has focussed on the mean and standard deviation (SD) of both coefficients in order to capture their behaviour and correlation with the input parameters. The numerical analysis has been defined through the use of GiD pre-processor tool [7]. An unstructured mesh has been created which enables a proper calculation of the lift and drag values through the definition of finer elements on the profile lines and a surrounding areas. The convergence has been ensured defining a proper amount of time steps. The numerical solver has been set up according to its internal definition; more detail can be obtained from reference [5].

Table 2 gives the list of the obtained minimum, maximum, SD, and mean value of the two output variables, $C_{l}$ and $C_{\mathrm{dp}}$ coefficients, for the eight defined cases.

The analyses of all these values have been used to confirm the known relationship between lift and drag, and AoA and $M$. These kinds of conclusions have led to the validation of the stochastic analysis tools used in this study.

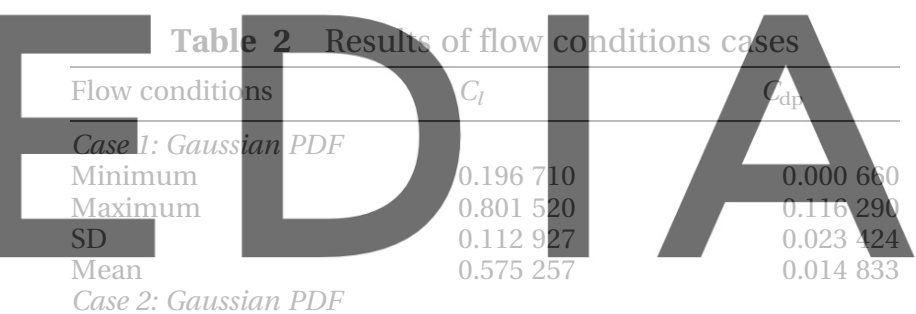

Case 2: Gaussian PDF

downinum the version w

SD

Mean

Case 3: Gaussian PDF

Minimum

Maximum

SD

Mean

Case 4: Gaussian PDF

Minimum

Maximum

SD

Mean

Case 5: Gaussian PDF

Minimum

Maximum

SD

Mean

Case 6: Gaussian PDF

Minimum

Maximum

SD

Mean

Case 7: Gaussian PDF

Minimum

Maximum

SD

Mean

Case 8: Uniform PDF

Minimum

Maximum

SD

Mean

Q.tsth

0.045442

0.563089

0.217150

0.887760

0.126863

0.581701

0.249090

1.031040

0.150591

0.578093

0.152880

1.049770

0.168275

0.565699

0.401550

0.861750

0.085143

0.562241

0.156320

0.871920

0.146843

0.559294

0.125770

0.844940

0.163701

0.465643
0.000812

0.001467

$-0.000300$

0.124900

0.027027

0.017832

$-0.000100$

0.103300

0.020772

0.014280

0.002500

0.137490

0.027084

0.016121

$-0.000100$

0.054020

0.008020

0.006400

0.000580

0.123860

0.031274

0.020944

$-0.000900$

0.124070

0.044329

0.033504 
Figures 1 and 2 show, respectively, the mean values of $C_{l}$ and $C_{\mathrm{dp}}$ for each case, together with their corresponding $\pm 3 \sigma$ range. A result with a wide $\pm 3 \sigma$ range means a big dispersion in the results obtained from the stochastic analysis and, consequently, a big dependence with respect to the corresponding stochastic input variable. On the other side, a short $\pm 3 \sigma$ range shows an almost insensitive output with respect the stochastic input value.

In Figs 1 and 2, the sensitivity of each output value can be analysed for each defined case. From these plots, one can immediately identify which one of the two coefficients is more affected by the variability of each input.

The comparison between the coefficient of variation of the input and the output values can also be used for the measurement of the dispersion in the obtained results. The coefficient of variation is defined as the ration between the mean deviation $(\sigma)$ and the mean value. Lower values of this coefficient mean lower dispersion in the stochastic variable. On the other side, if both the input and the output variables have the same coefficient of variation, it means that the output has exactly the same variability (dispersion of values) as the input. This implies that the variability of the input is directly transferred to the output neither without adding additional dispersion nor without reducing it.

In Table 3, coefficients of variation for both inputs, AoA and $M$, and for both outputs, $C_{l}$ and $C_{\mathrm{dp}}$, are tabulated. It is easy to realize that $C_{\mathrm{dp}}$ distribution is more sensitive to input variability than $C_{l}$. Comparing coefficients of variation obtained when $M$ or AoA are constant (cases 1 and 2). $M$ produces bigger effect than AoA, which in both $C_{l}$ and $C_{\mathrm{dp}}$ distributions present lower coefficients of variation.

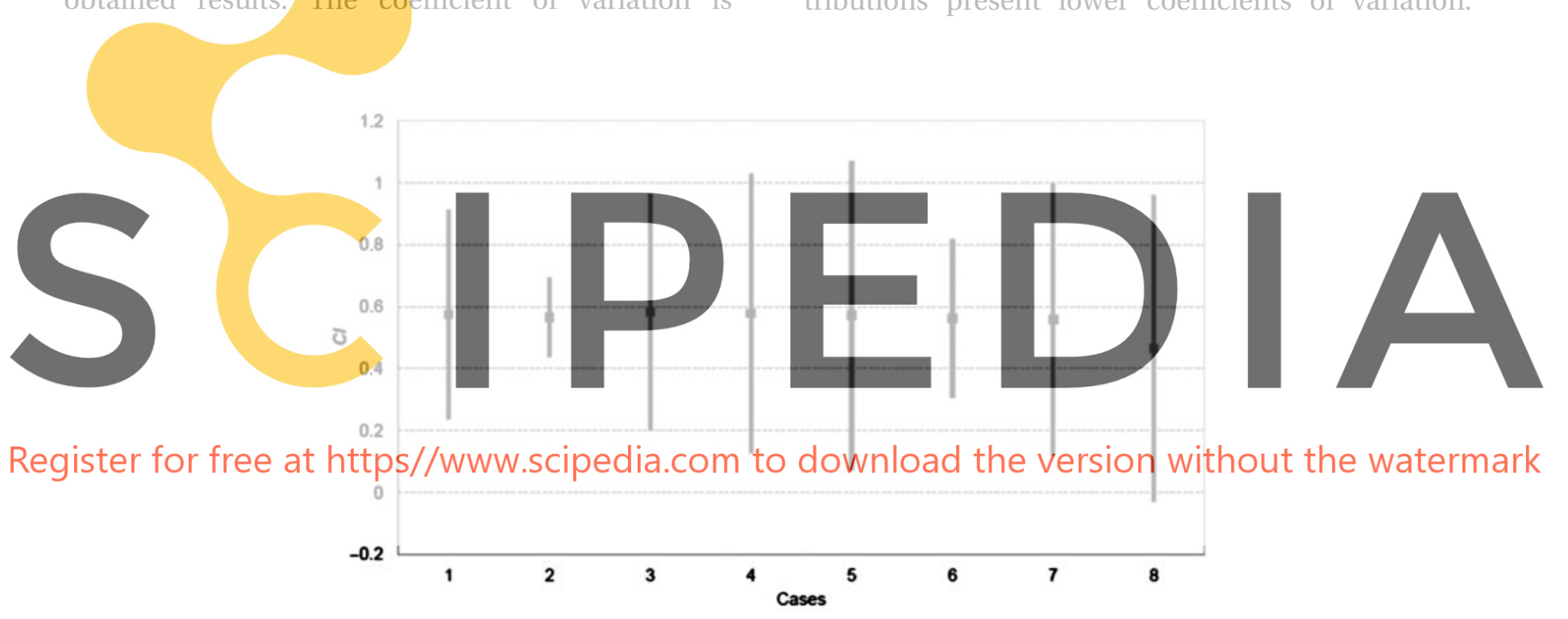

Fig. 1 Mean values and SD ranges for $C_{l}$

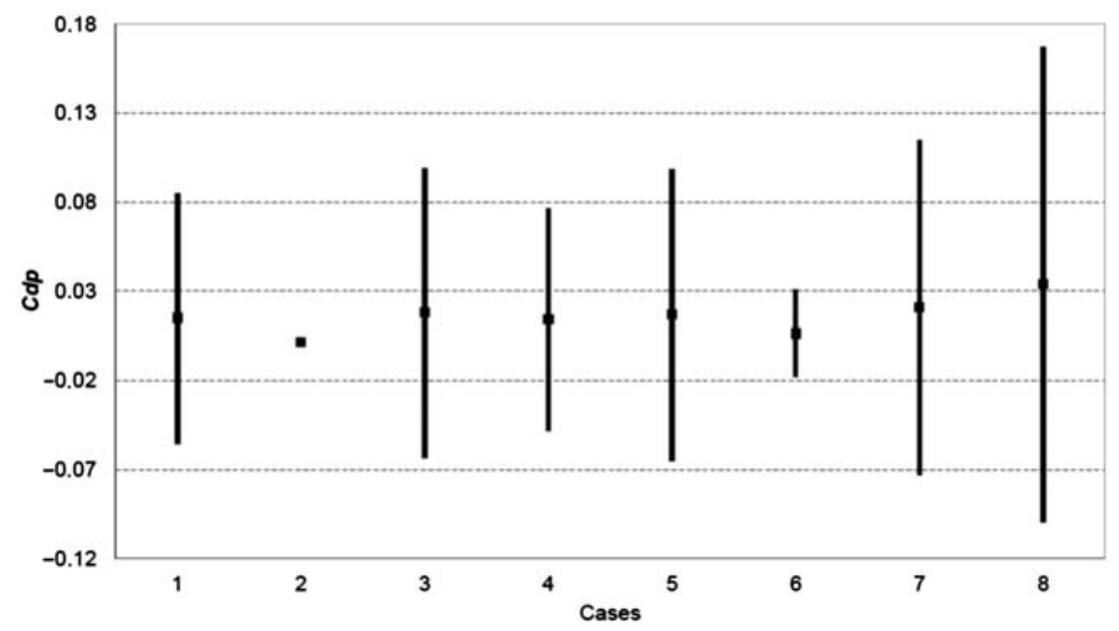

Fig. 2 Mean values and SD ranges for $C_{\mathrm{dp}}$ 
Other cases present a clear difference between coefficient values for $C_{l}$ and $C_{\mathrm{dp}}$, while coefficient values for $C_{l}$ cases are lower than 1 , around 0.25 as mean. Values for $C_{\mathrm{dp}}$ case are usually higher than 1, around 1.4 as mean. One can realize how far are coefficients of variation of input values and those obtained for output values. Coefficient of variation for $C_{l}$ is pretty close to the original ones for AoA and $M$, but $C_{\mathrm{dp}}$ does

Table 3 Coefficients of variation

\begin{tabular}{lllll}
\hline Flow conditions & AoA & $M$ & $C_{l}$ & $C_{\text {dp }}$ \\
\hline Case 1: Gaussian PDF & 0 & 0.114 & 0.196 & 1.579 \\
Case 2: Gaussian PDF & 0.125 & 0 & 0.081 & 0.553 \\
Case 3: Gaussian PDF & 0.125 & 0.114 & 0.218 & 1.516 \\
Case 4: Gaussian PDF & 0.25 & 0.114 & 0.260 & 1.455 \\
Case 5: Gaussian PDF & 0.375 & 0.114 & 0.297 & 1.680 \\
Case 6: Gaussian PDF & 0.125 & 0.057 & 0.151 & 1.253 \\
Case 7: Gaussian PDF & 0.125 & 0.171 & 0.263 & 1.493 \\
Case 8: Uniform PDF & 0.048 & 0.060 & 0.352 & 1.323 \\
\hline
\end{tabular}

not present the same behaviour with a higher coefficient of variation compared with original ones for input variables.

In addition to the previous analysis, which uses coefficient of variation as the main comparing criteria, the following figures provide a graphical analysis of the variability. They present the behaviour of each output compared with the input. Figures 3 and 4 show $C_{l}$ and $C_{\mathrm{dp}}$ versus $M$. The results of all the analyses performed for all eight stochastic defined cases are plotted. In the same way, in Fig. $5, C_{l}$ is plotted versus AoA.

In Fig. 3, it can be realized how $C_{l}$ increases with $M$, up to a limit, where shock waves begin to appear. It follows the typical shape of a polar curve but, in this case, $M$ is following a Gaussian distribution. Values are spread around the mean value, with a bigger density at this value and reducing it as values are far from

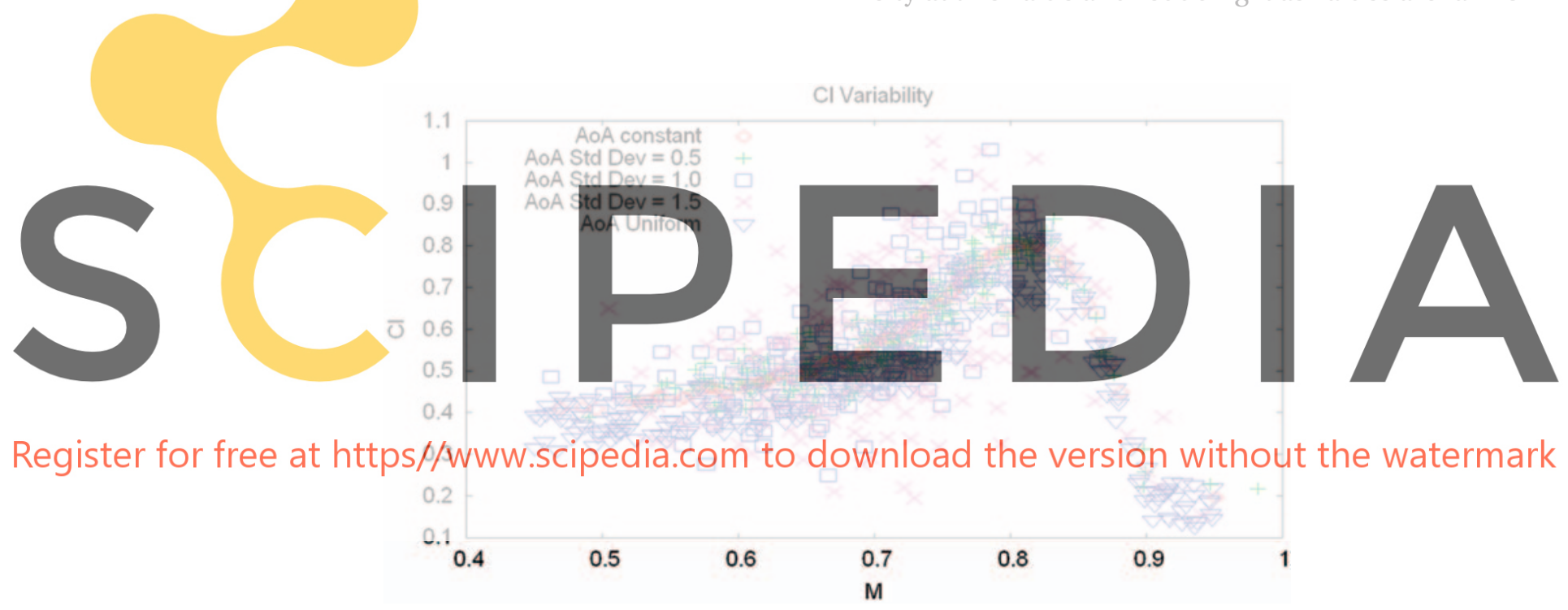

Fig. $3 C_{l}$ vs $M$ with several distributions of AoA

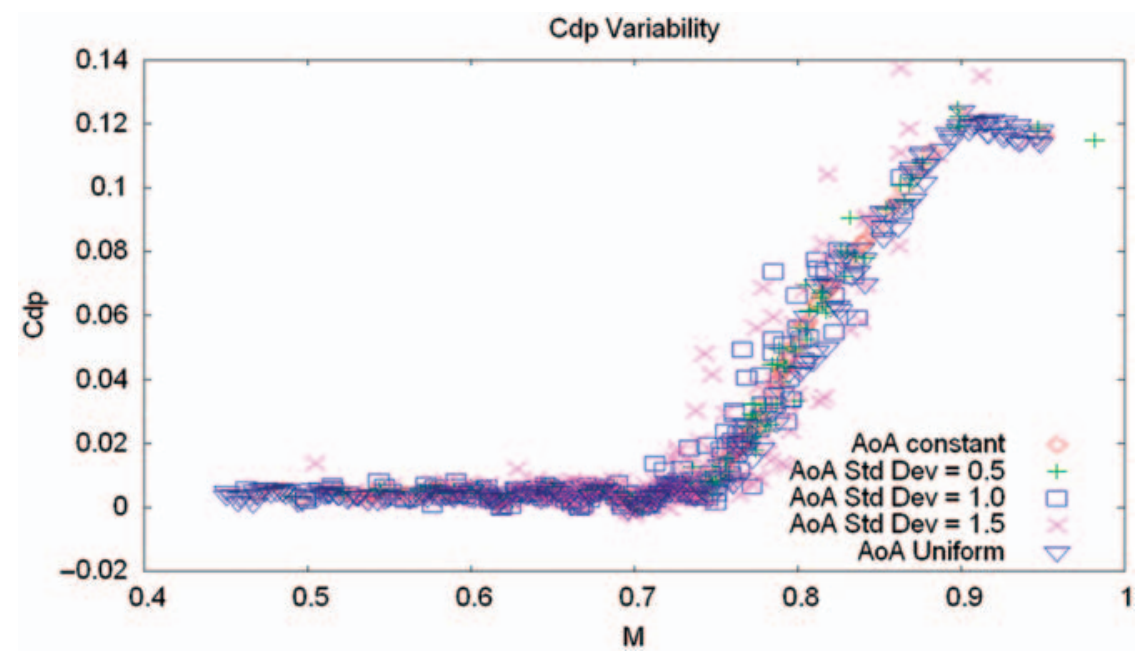

Fig. $4 C_{\mathrm{dp}} v s M$ with several distributions of AoA 
the mean value. In addition, it shows a clear dependency between $C_{l}$ and the AoA: on one hand, in subsonic regime, $M<0.8$, the plot presents a big variability of $C_{l}$ while increasing the SD of the AoA, even considering the effect of doubling and multiplying by 3 this value, or considering a uniform distribution. On the other hand, in transonic regime, the variability (dispersion of values) of $C_{l}$ is lower, and the plot is a thinner line of points.

In Fig. 4, pressure drag coefficient, $C_{\mathrm{dp}}$, shows a quite constant behaviour until transonic values of $M$ start to increase. The CFD solver used, PUMI, only provides pressure drag values, because it is based on Euler equations, without calculating boundary layer effects. Then, the value provided for $C_{\mathrm{dp}}$ is negligible up to transonic values, when a shock pressure appears and $C_{\mathrm{dp}}$ produces significant values.

Figure 4 presents a lower variability of $C_{\mathrm{dp}}$ due to the variability of the AoA. In opposite to what happens with $C_{b}, C_{\mathrm{dp}}$ variability increases in transonic regime due to the presence of shock waves. Variability in subsonic regime is mainly due to numerical errors of the solver more than to $C_{\mathrm{dp}}$

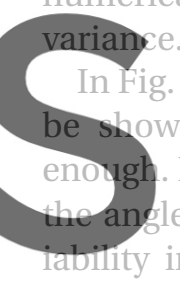

5 , a straight line portion wn because the values . Figure 5 shows a lineart e of attack and $C_{b}$, as it is

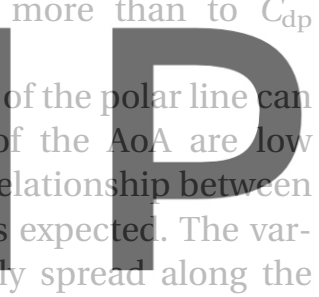

curve without breaking the linear relationship Registerefor, freelat.https//www.scipedia.com to In all the above figures, the effect of the statistical distributions applied to $M$ or AoA can be detected. In

Figs 3 and 4, the density of values is bigger around $M=0.734$, which is the mean value of the $M$. On the other side, Fig. 5 shows that the variability of the AoA produces thicker plots that increase their thickness when increasing the SD of this angle.

Output variability has been analysed for the RAE2822 airfoil case. The obtained results follow the expected trends. It can be concluded that the input variable producing the biggest dispersion of values in the output parameters is the Mach number.

These analyses provide information of how lift and drag are affected by variability in the flow conditions; namely angle of attack and Mach number. Not only can the well-known relationship between them be identified on the plots, but also the combined effect of both variables at the same time, and for several statistical distributions. Obviously, the best representation of this relationship can be seen when one of the variables remains constant, but it can also be identified when the variability effects appear in the plot, even if the plot becomes thicker (disperse) due to the representation of variability.

The stochastic procedure has performed well in a CFD environment. The use of the coefficient of variation has been demonstrated as mandatory in order to compare the dispersion of results with the disper-
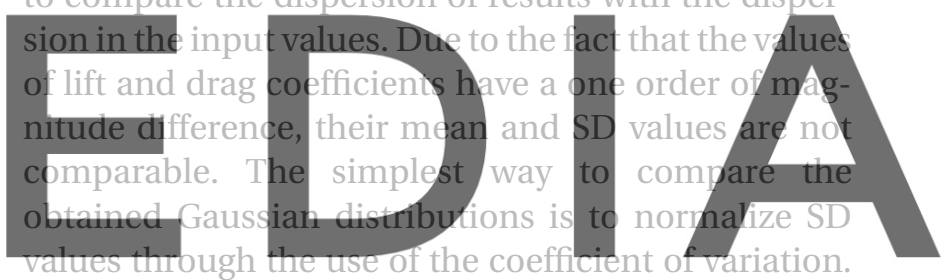

The fact that PUMI CFD solver is based on Euler dQw/ridoad the eversioin tovithoiduthien.wateremark the calculation of drag is an approximation that can affect the final behaviour of the results. This fact demonstrates the need of knowing the solver and understanding its use. If not, it can easily lead to wrong conclusions.

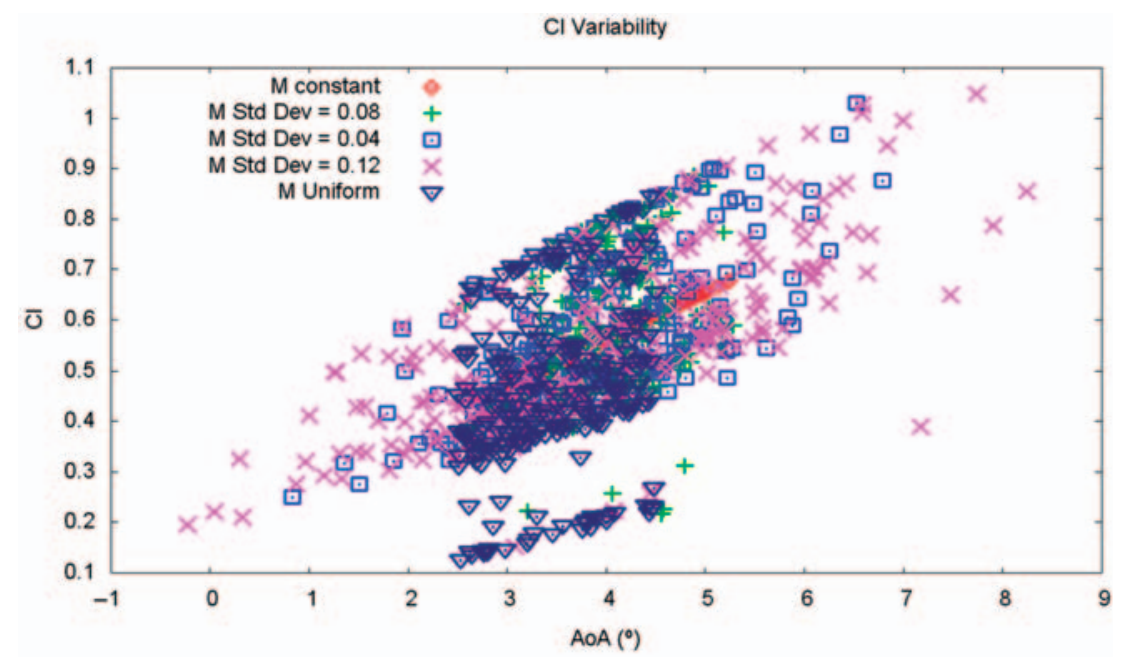

Fig. $5 C_{l}$ vs AoA with several distributions of $M$ 
STAC enables the definition of samples using both Monte-Carlo and Latin Hypercube techniques. Several tests have been performed and they lead to the conclusion that both sampling techniques provide similar results. This conclusion is due to the fact that the comparison analysis uses mean values as its main target, and it is well known that Latin Hypercube improves the covariance convergence compared to Monte-Carlo method, but not necessarily the mean value.

\subsection{Example of a stochastic aero-elastic analysis}

In a similar way in the stochastic CFD analysis, an aero-elastic problem has been defined. The RAE2822 profile is, again, the baseline geometry. An uncertainty definition of both flow and structural input parameters has been used. The flow field parameters are the Mach number and the angle of attack. The structural parameters are the damping coefficients for the vertical and the angular movements, and the $x$-coordinate of the elastic axis. All of them have been stochastically defined using a Gaussian statistical distribution (Table 4). For each stochastic analysis, a set of 200 samples or shots have been calculated using the Latin Hypercube sampling technique.

Three different types of analysis have been defined; the first one is a deterministic analysis that uses the mean values of all parameters as input values without considering their stochastic nature. This first analysis has been defined to be used as a reference (case 0). The second analysis considers a simultaneous stochastic behaviour of all input parameters (case 1). The third analysis is, in fact, a set of separate stochastic cases. In each of these cases, only one of the five input parameters has been considered as stochastic (cases $2 \mathrm{a}-\mathrm{e}$ ), whereas the rest have been maintained as in the deterministic case. The total number of cases is equal to the number of input parameters for which a stochastic nature has been considered (Table 4).

An in-house aero-elastic code based on particle

finite-element method [8, 9] has been used as the

Table 4 Analysed cases for the ensitivity study

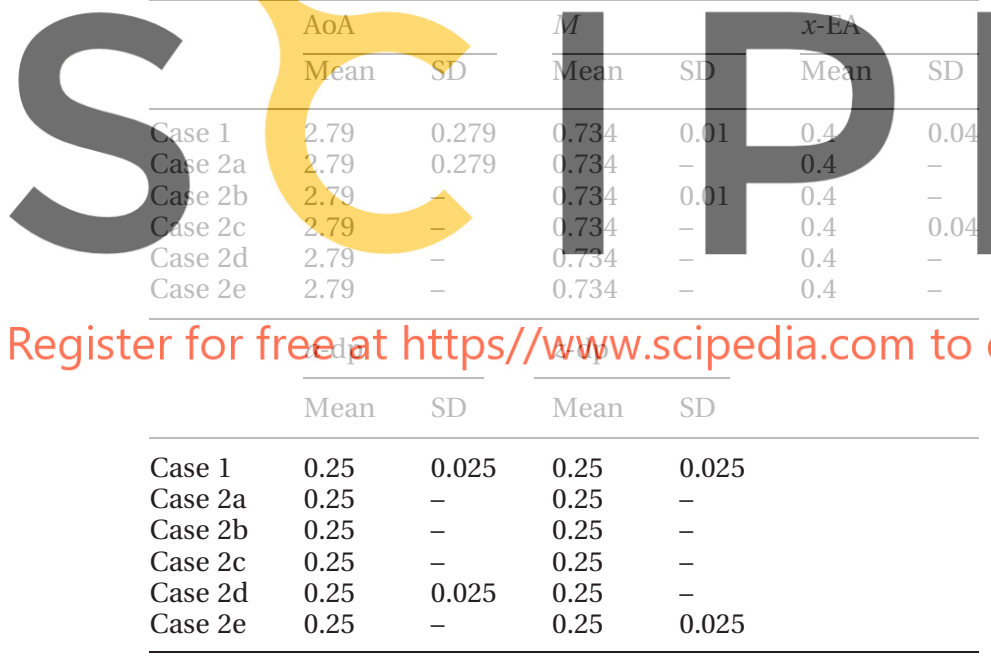

main analysis tool. It rakes advantage of Eyler cquations for flow calculations, coupled with t twodegiees-of-freedom pitch and plunge) structural
model for the elasticity model. The selected output is theta, $\theta$, whic ter plien which is the angular oscillation of the flut-

Figures 6 to 12 show a comparison between the

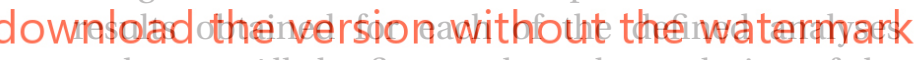
and cases. All the figures show the evolution of the angular spin angle of the profile through time. The figures are used to compare the effects when introducing variability to some of the design variables. Figure 6 shows the results for the deterministic case. In this case, the elastic behaviour of the wing produces a first oscillation, which decreases in $0.45^{\circ}$ the

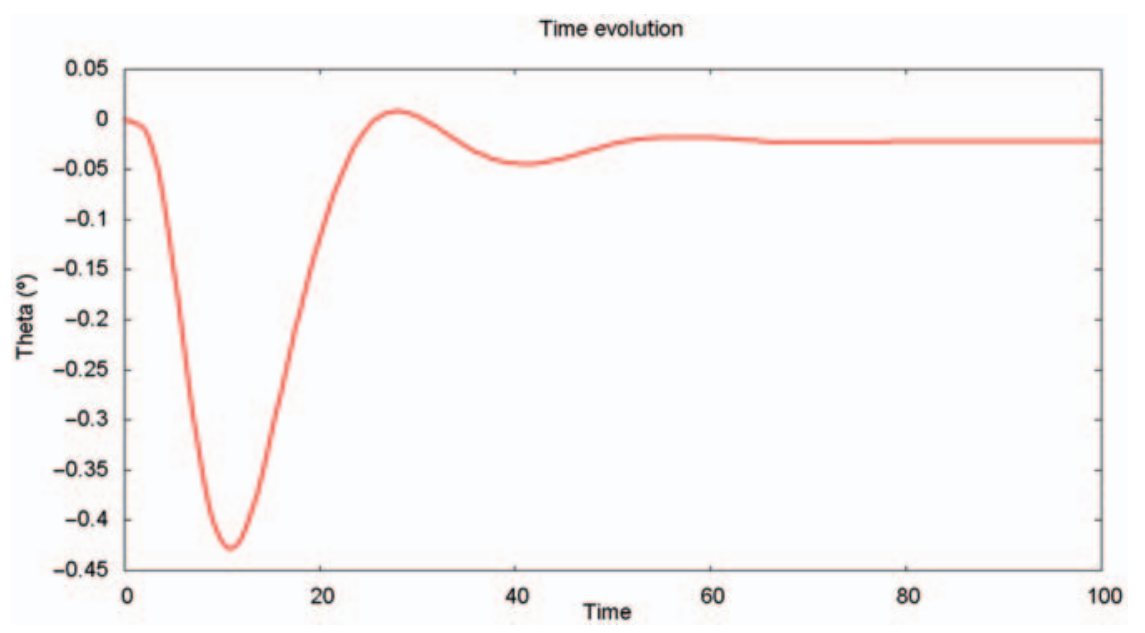

Fig. 6 Time evolution of angular oscillation applying nominal deterministic values 


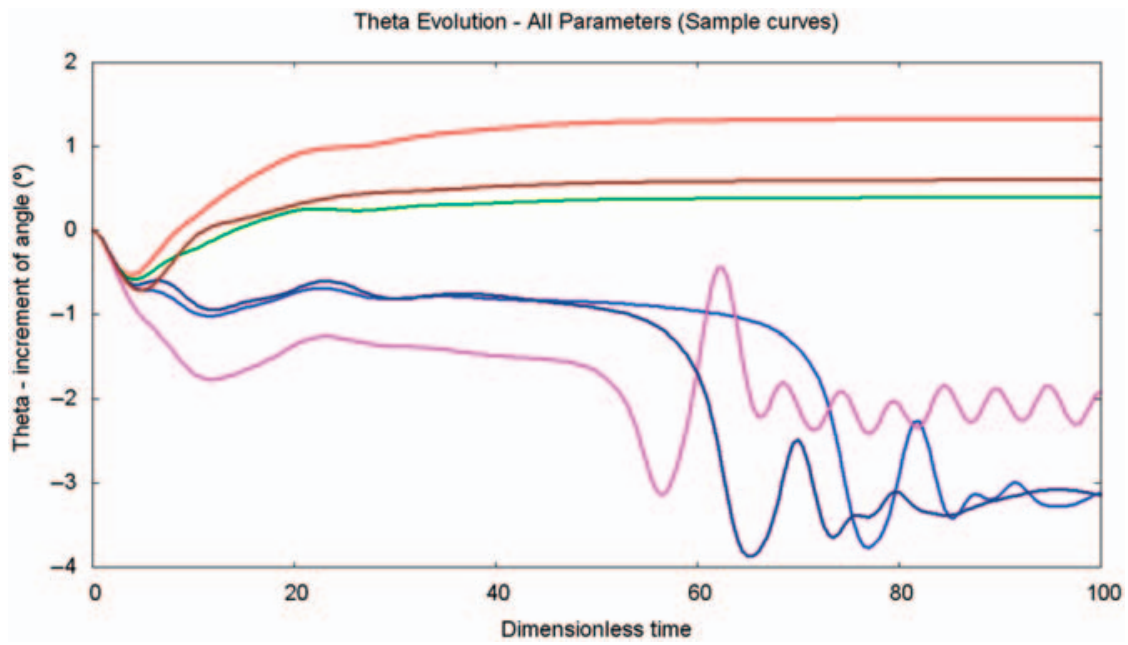

Fig. 7 Time evolution of angular oscillation applying stochastic definition of all parameters
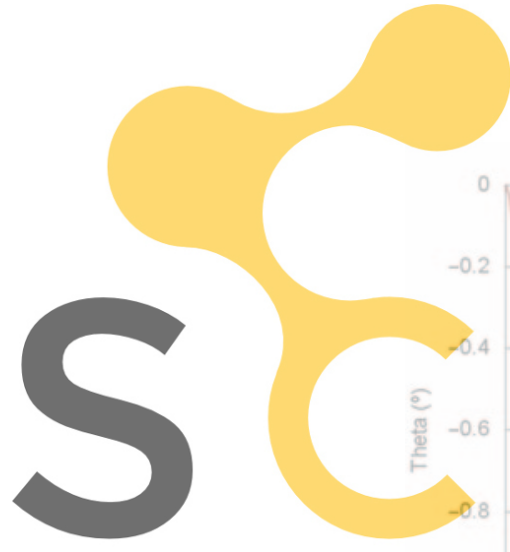

Theta Evolution - Variation of AoA (Sample curves)
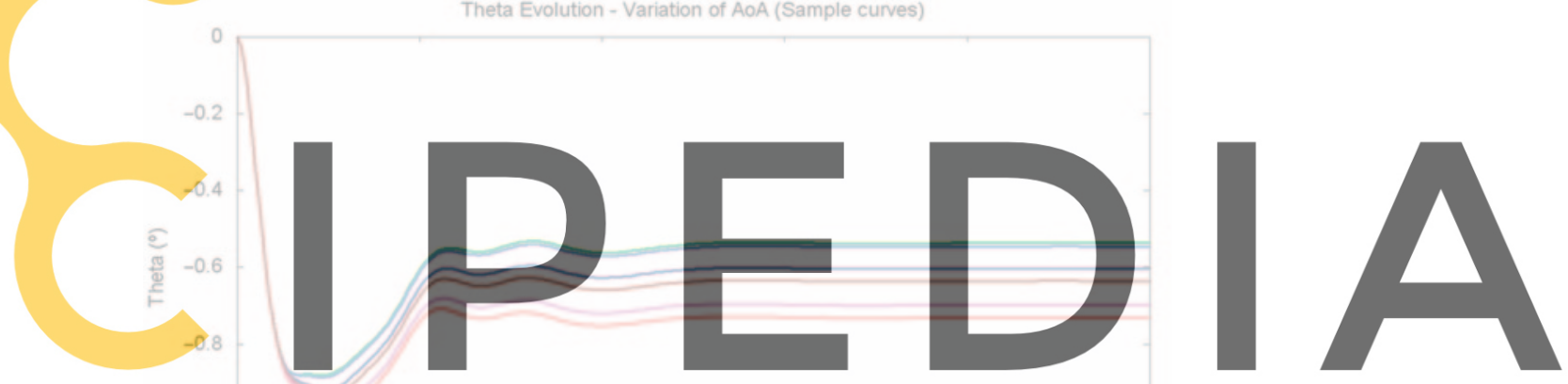

Register for free at https//www.scipedia.com to download the version without the watermark $-1.2$
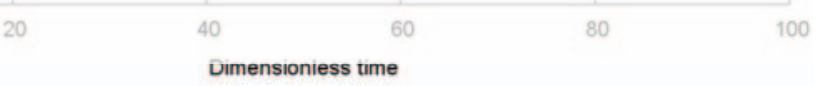

Fig. 8 Time evolution of angular oscillation applying stochastic definition to AoA

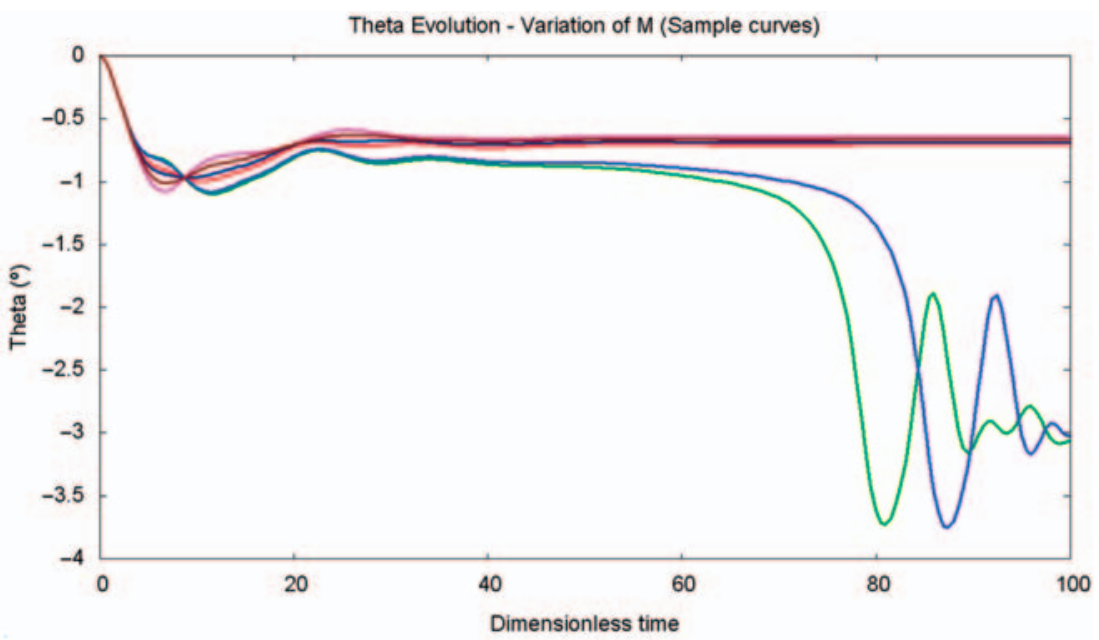

Fig. 9 Time evolution of angular oscillation 


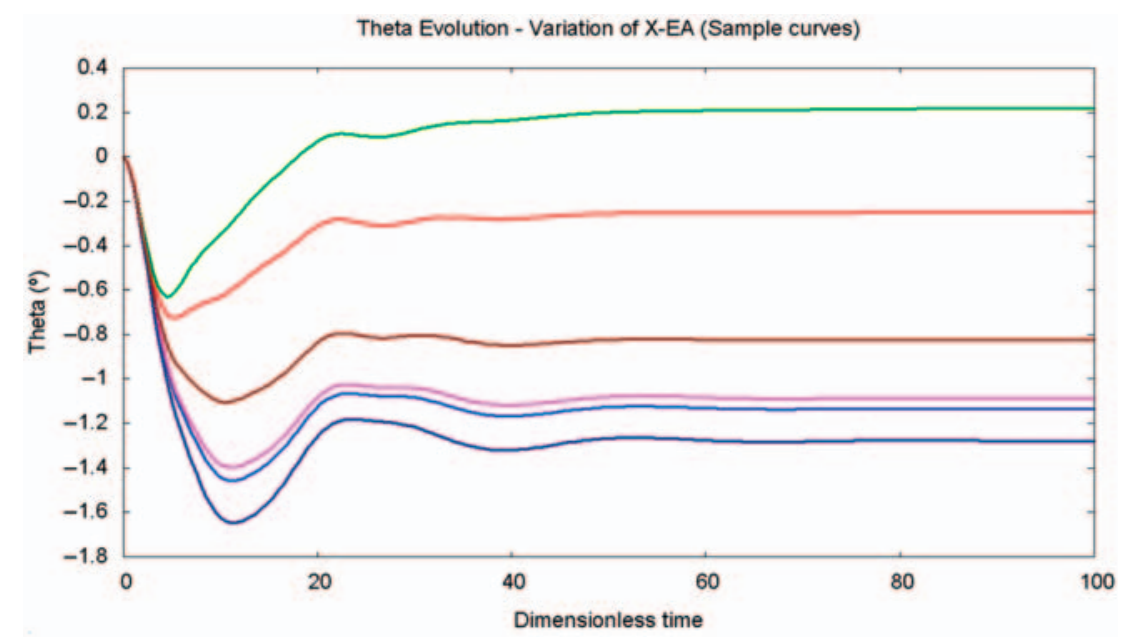

Fig. 10 Time evolution of angular oscillation applying stochastic definition to $x$-EA
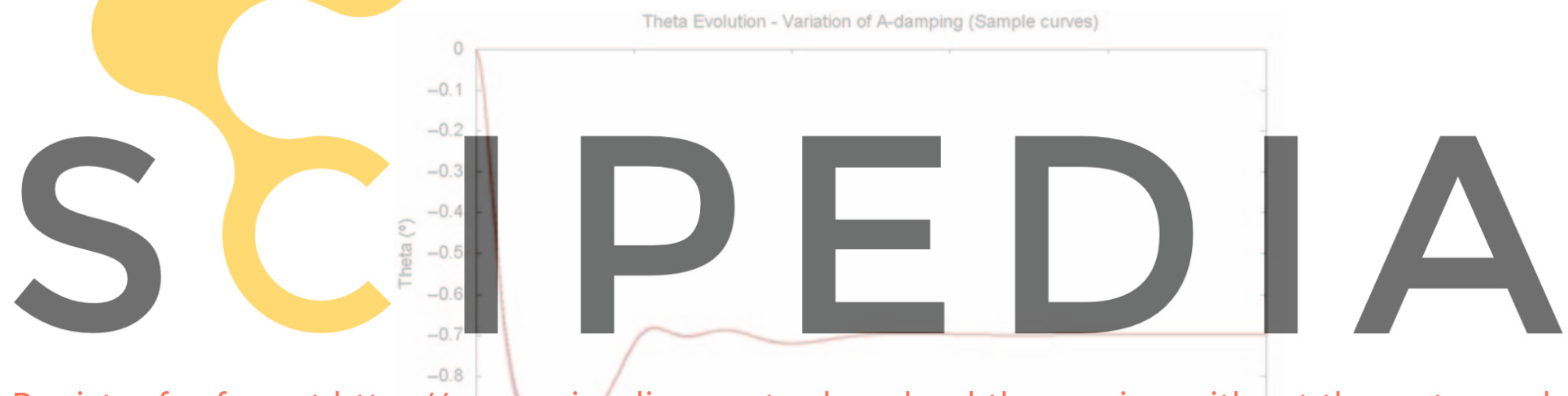

Register for free at https/fwwww.scipedia.com to download the version without the watermark

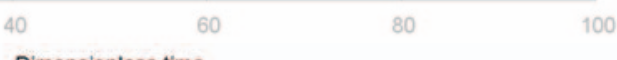

Dimensionless time

Fig. 11 Time evolution of angular oscillation applying stochastic definition to $z$-dp

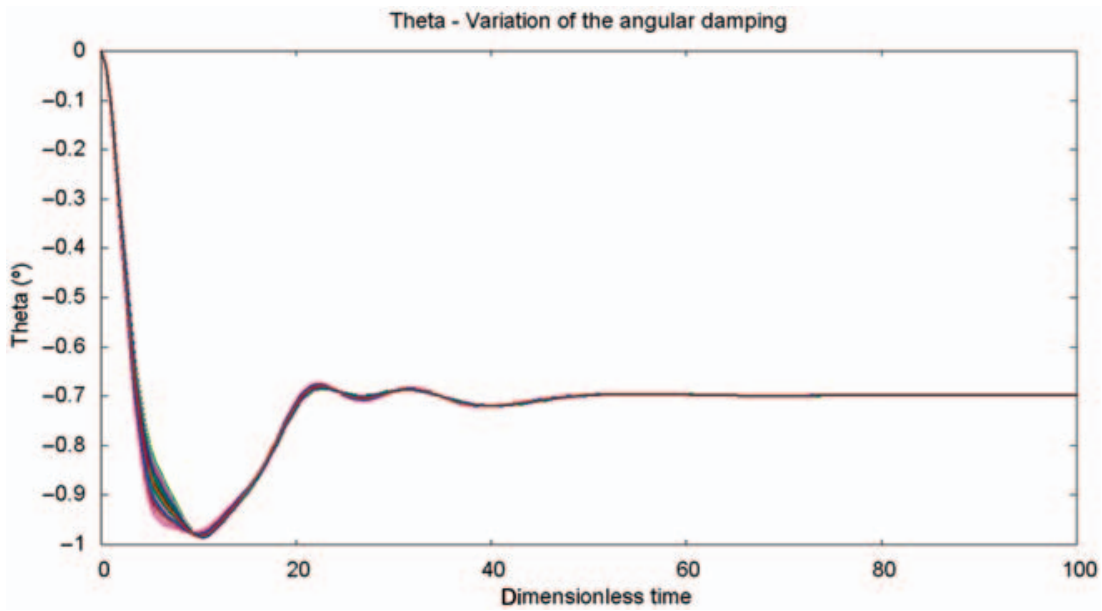

Fig. 12 Time evolution of angular oscillation applying stochastic definition to $a$-dp 
actual angle of attack, but after that point the flow stabilizes around the profile and one can consider that the actual angle of attack remains constant at $0.021^{\circ}$ below the nominal value. Figure 7 shows some samples among the whole set of curves from the profiles corresponding to the second analysis, when all the parameters are stochastically defined in a simultaneous way. It shows a set of plots that represent all the analysed cases. The first important point to remark in the new set of results is that this plot follows a similar pattern to the deterministic case. The shape and behaviour of the curves in Fig. 7 are similar to those shown in Fig. 6. The second important remark to be done is the flutter phenomenon that appears in some cases. After an apparent trend leading to convergence, some values of the stochastic parameters make the convergence loose and oscillation grows with time. This flutter phenomenon, which is an undesired structural effect, is produced by some of the combinations of values of input parameters.

In order to detect which of the stochastic parameters induces the flutter, a separate stochastic analysis for each input variable has been perforned. Figure 8
shows some sample curves of the time evolution of
the-escillation when only angle of attack is stochasti-
cally defined. The first oscillation is bigger than that
obtaned in the deterministic case. Howgver, the final
convergence value is lower. Anyway, it can be easily realized that all the analysed cases converge to a

Registeder forlefpee at https//www.scipedia.com On the other hand, Fig. 9 shows some of the results corresponding when the Mach number is stochastically defined. Now, the lack of convergence of some samples is easily detected. The time evolution does not follow the same pattern of other analyses.

Figures 10 to 12 show some of the results corresponding to the stochastic definition of $x$-EA, $z$-dp, and $a$-dp, respectively. In all the cases, the evolution of the angular oscillation converges to a stable value. Figure 11 shows how the vertical damping coefficient produces a very small variability value, even plotting only few samples. The stochastic range of values of this coefficient is small enough to ensure a tight variability range of the oscillation angle. It is easily understandable that the damping coefficient of the vertical movement does not directly affect the oscillation angle.

Both damping coefficients present a very narrow set of curves (Figs 11 and 12). In the first case, the vertical movement damping has a low effect on the variability of theta angle $(\theta)$, as it could be guessed $a$ priori. What was hard to predict is that angular damping coefficient has also a tight variability effect. SDs of both damping coefficients are about 10 per cent of the mean value, which are similar values to those defined for other parameters. However, in Table 6, one can realize their low effects in the variability of the output.

Table 4 gives a brief summary of all analysed cases and the applied values in each one. Both damping coefficients have smaller SD, which leads to the obtained results.

Each of these cases has been used to analyse the behaviour of additional output parameters, like the vertical movement of the profile, and the aerodynamic coefficients of lift, drag, and momentum.

Other output variables, like aerodynamic coefficients (lift, drag, and momentum), or structural parameters like vertical deformation of the wing can be analysed in the same way as it has been done with $\theta$, the angular spin of the profile. The description of the results has been focused on $\theta$ in order to simplify the presentation. However, similar conclusions can be taken from other output parameters.

Results can be summarized using the coefficient of variation obtained for all the analyses. In Table 5, the obtained coefficients are listed.

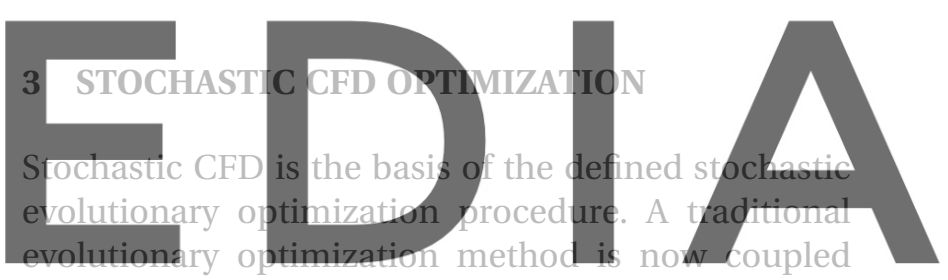

with the stochastic tool enabling the stochastic anal-

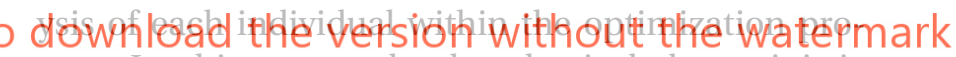
cess. In this approach, the classical deterministic analysis of each individual is substituted by a complete stochastic analysis. The stochastic analysis of a given individual provides now a cloud of points, and the fitness function is computed through the mean value of the output, which is used as the stochastic fitness. The mean deviation of the output can also be used as a measure of the robustness of the

Table 5 Coefficients of variation

\begin{tabular}{llllll}
\hline & AoA & $M$ & $x$-EA & $a$-dp & $z$-dp \\
\hline Case 1 & 0.0927 & 0.0133 & 0.0980 & 0.0960 & 0.0930 \\
Case 2 & 0.0972 & 0.0 & 0.0 & 0.0 & 0.0 \\
Case 3 & 0.0 & 0.0130 & 0.0 & 0.0 & 0.0 \\
Case 4 & 0.0 & 0.0 & 0.1084 & 0.0 & 0.0 \\
Case 5 & 0.0 & 0.0 & 0.0 & 0.1069 & 0.0 \\
Case 6 & 0.0 & 0.0 & 0.0 & 0.0 & 0.0981 \\
\hline & \multirow{2}{*}{ Th (min) } & $h / c(\max )$ & $C_{\mathrm{d}}(\mathrm{min})$ & $C_{l}(\mathrm{~min})$ & $C_{m}(\mathrm{~min})$ \\
\hline Case 1 & -0.6952 & 0.1332 & -0.7412 & 10.2331 & -0.6719 \\
Case 2 & -0.0487 & 0.0502 & -0.2456 & 0.0602 & -0.0376 \\
Case 3 & -0.6854 & 0.0120 & -0.3253 & 7.0153 & -0.6480 \\
Case 4 & -0.3362 & 0.1583 & -0.4269 & 0.1032 & -0.3340 \\
Case 5 & -0.0077 & 0.0223 & -0.0103 & 0.0094 & -0.0061 \\
Case 6 & -0.0018 & 0.0026 & -0.0633 & 0.0041 & -0.0038 \\
\hline
\end{tabular}


design compared with the variability of the input value $[10,11]$.

A traditional CFD stochastic analysis requires a significant amount of different analysis, and the corresponding global computational cost can be very expensive. This cost can be much more prohibitive when a stochastic analysis is performed for each of the different designs obtained during an evolutionary optimization process. This justifies the use of a surrogate model. In this study, the use of artificial neural networks (ANN) have been selected because of its capability to deal with a vast type of different problems [12-15]. It will be integrated in the evolutionary algorithm in parallel with the stochastic CFD tool.

Based on a proved evolutionary algorithm, like NSGA-II [16-18], some tests have been performed in order to show the capabilities of the proposed methodology. The parameters used to set up the genetic algorithm are as follows:

(a) population size: 16 ;

(b) number of generations: 100;

(c) probability of cross-over: 0.99 ;

(d) probability of mutation: 0.25 ;

\subsection{Deterministic optimization problem}

As an illustration example, the results corresponding to a deterministic multi-objective optimization problem are first shown. The problem is defined as

Minimize

$$
\begin{aligned}
& f_{1}=\frac{1}{C_{l}} \\
& f_{2}=C_{\mathrm{dp}}
\end{aligned}
$$

For a profile defined using Bezier curves, which takes RAE2822 coordinates as starting point (Fig. 13).

Table 6 shows the constraints applied to the coordinates of the knot points of the Bezier curves used for the geometrical parametrization of the problem.

In order to compare the solutions obtained by a deterministic optimization problem and a stochastic one, the deterministic problem has been first solved using the following values for the Angle of attack (AoA) and the Mach number $(M)$

$$
\begin{aligned}
& \text { Angle of attack, AoA }=2.79 \\
& \text { Mach number, } M=0.734
\end{aligned}
$$

The NSGA-II optimizer and ANN surrogate model have been used. The following parameters have been defined to set up the algorithm:

(a) population size: 24 ;

(b) number of generations: 300 ;

(c) probability of cross-over: 0.95 ;

(d) probability of mutation: 0.166 667;

Figure 14 shows the obtained population and Pareto front as the solution of the deterministic optimization problem.

\subsection{Integrating stochastic CFD and evolutionary algorithms}

The deterministic example has been transformed into a stochastic one, coupling the stochastic CFD analysis and the evolutionary algorithm. The problem now becomes

Minimize

$$
\begin{aligned}
& f_{1}=\operatorname{mean}\left(\frac{1}{C_{l}}\right) \\
& f_{2}=\operatorname{mean}\left(C_{\mathrm{dp}}\right)
\end{aligned}
$$

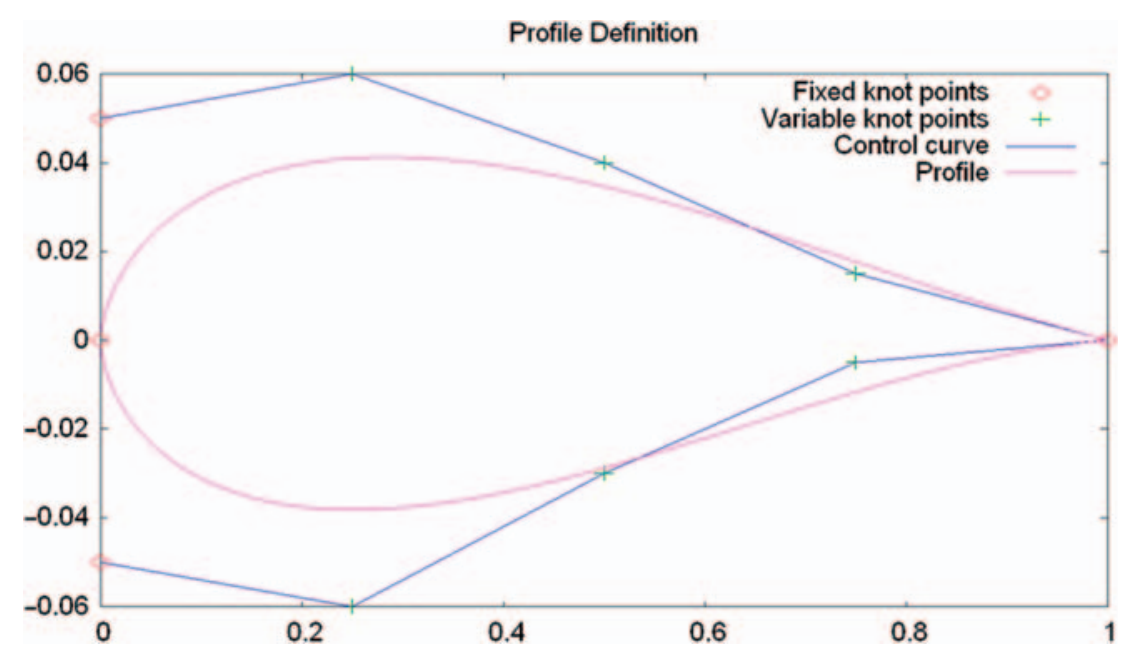

Fig. 13 Bezier curves and knot points under EA control 
For a profile defined using Bezier curves, which takes RAE2822 coordinates as starting point, and same as Fig. 13. The values of Table 6 are still used for the constraints applied to the coordinates of the knot points of the Bezier curves used for the geometrical parametrization of the problem.

Table 7 shows the mean values and the SD corresponding to a Gaussian PDF definition for AoA and $M$. Mean values are the same as the deterministic values previously defined. Using Monte-Carlo method, a single set of 250 samples have been generated to model the input parameters, and it has been used for the analysis of all individuals.

The proposed integration of the stochastic analysis and the evolutionary algorithms is defined as follows.

1. Initial population:

(a) define individuals using geometrical variables;

(b) calculation of the fitness of each individual using a stochastic analysis; obtain a set of samples to calculate mean and variance of the fitness.

2. Evolutionary algorithm; main loop:

(a) generate new populations of geometries; selection, cross-over, and mutation of individuals;

Table 6 Geometrical constraints

\begin{tabular}{lllll}
\hline & $\begin{array}{l}X \\
\text { coordinate }\end{array}$ & $\begin{array}{l}Y \\
\text { coordinate }\end{array}$ & $\begin{array}{l}\text { Lower } \\
\text { bound }\end{array}$ & $\begin{array}{l}\text { Upper } \\
\text { bound }\end{array}$ \\
\hline Knot x1s, y1s & 0 & 0 & - & - \\
Knot x2s, y2s & 0 & 0.05 & - & - \\
Knot x3s, y3s & 0.25 & Variable & 0.05 & 0.085 \\
Knot x4s, y4s & 0.5 & Variable & 0.03 & 0.06 \\
Knot x5s, y5s & 0.75 & Variable & 0.01 & 0.02 \\
Knot x6s, y6s & 1 & 0 & - & - \\
Knot x2l, y2l & 0 & -0.05 & - & - \\
Knot x31, y31 & 0.25 & Variable & -0.06 & -0.03 \\
Knot x41, y41 & 0.5 & Variable & -0.035 & -0.02 \\
Knot x5l, y5l & 0.75 & Variable & -0.015 & -0.005 \\
\hline
\end{tabular}

(b) calculation of the fitness of each individual using the stochastic analysis.

3. End of iterations:

(a) reach of convergence criteria;

(b) reach of maximum time or number of populations.

The use of a surrogate model is almost mandatory to reduce the computational cost of the whole process. The used ANN has been trained in order to provide results with less than 1 per cent of error.

In order to ensure ANN feasibility, the Pareto fronts obtained using the direct analysis tool and the ANN have been compared. Figure 15(a) shows the difference between the whole populations obtained in both cases. Figure 15(b) shows both Pareto fronts and the difference existing between them. This difference remains below a 3 per cent which has been considered as acceptable.

Figure 16 shows a comparison between the deterministic and the stochastic optimization solutions. Both of them use the same problem definition except for AoA and $M$, which are the stochastic variables. Both use ANN coupled with the optimizer.

One should notice that the shapes of both fronts are similar, but one front is displaced with respect to the other one. The stochastic front is clearly forwarded, as it can be seen in the amplified picture (Fig. 16). Solutions are different because the stochastic solution is dealing with the mean of a cloud of evaluations instead of a single value as the deterministic case is. Some of these points are affected by the presence of a shock wave, whereas the deterministic optimization does not take into account the possibility of having this shock wave. From this point of view, stochastic definition produces a more robust solution.

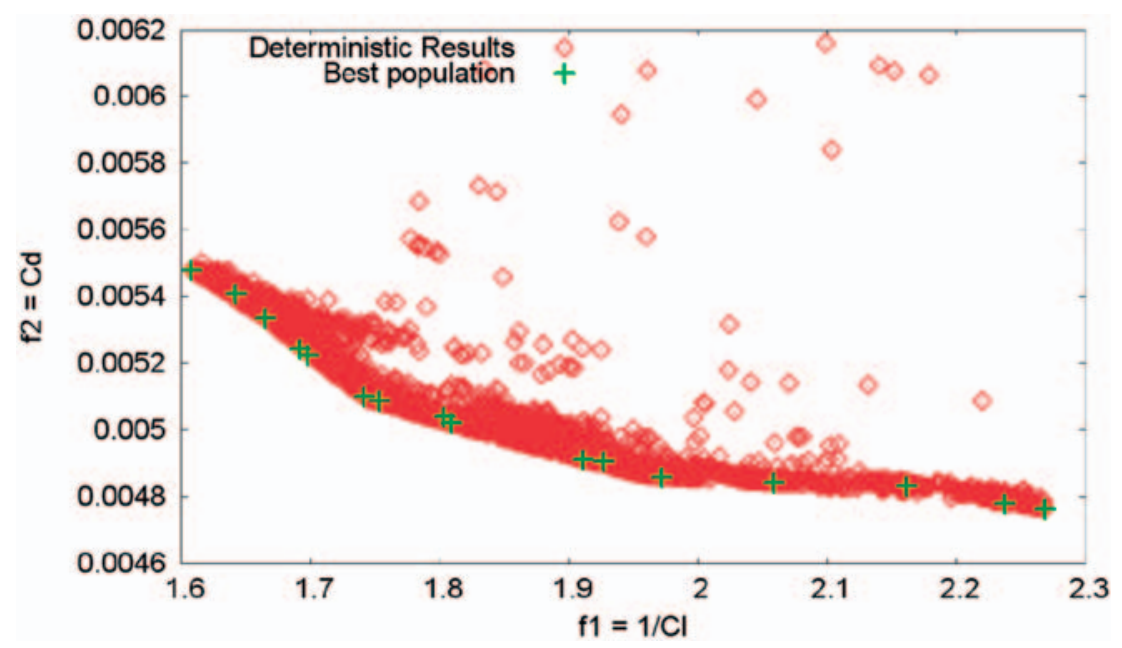

Fig. 14 Deterministic multi-objective optimization 
Closest Pareto fronts could be obtained in low subsonic situations, but when work with transonic flow fields the differences are relevant.

\subsection{Applying statistical input behaviour to the optimization process}

In order to simplify the process, the previous stochastic optimization process was defined using the same set of stochastic values for AoA and $M$ for all the analysed individuals. This is, in fact, an extreme case of a multi-point optimization problem with a very high number of points. Anyway, a multi-point evaluation of all the individuals is not really representative of the statistical representation of the input variables and the next step has been to really consider the generation of a different set of random input variables for the analysis of each individual.

Table 7 Stochastic constraints

\begin{tabular}{lll}
\hline & Mean & SD \\
\hline Angle of attack & 2.79 & 0.279 \\
Mach number & 0.734 & 0.05 \\
\hline
\end{tabular}

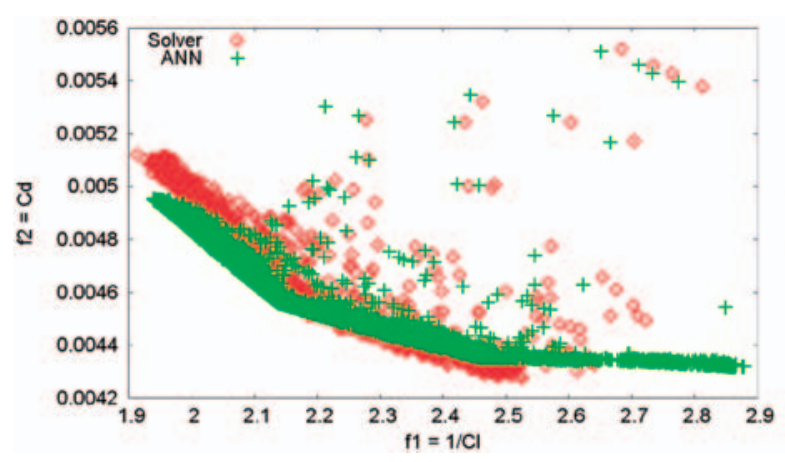

It could be considered that using a single random input definition for the individuals analysed during the whole optimization process is enough (multipoint approach). Nevertheless, the statistical definition of the input variables mainly intends to capture its random behaviour. A different sampling for each individual introduces an additional variability that increases the robustness of the result. Hence, random samples are now generated for each generation and individual, it is the so-called variable random definition.

Figure 17 shows the comparison between the results obtained with the fixed random definition and those obtained with a different random definition for each individual. It can be detected how the fixed definition also fixes the front of the solution, clearly defining a linear trend, while the variable definition breaks this regularity. It means that the variable definition affects the evaluation of the fitness function enabling to capture better results.

If attention is focused only in the shape of the Pareto fronts, Fig. 18 shows how optimal results for the fixed definition lead to a poor number of individuals compared with those existing in the Pareto front

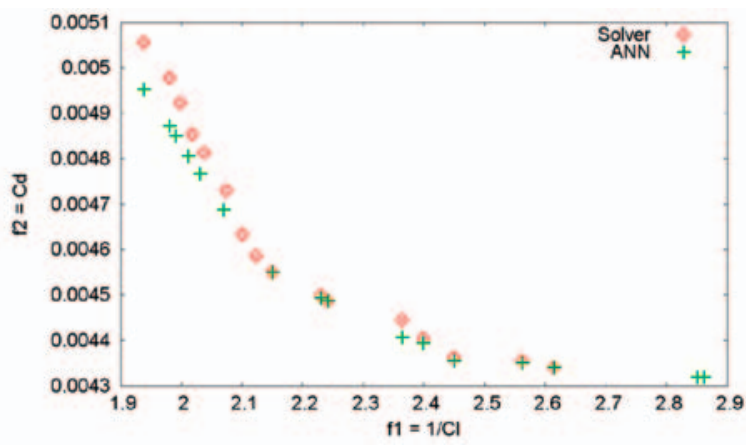

Fig. 15 Solution of the optimization using the analysis tool and ANN: (a) whole population and (b) Pareto front

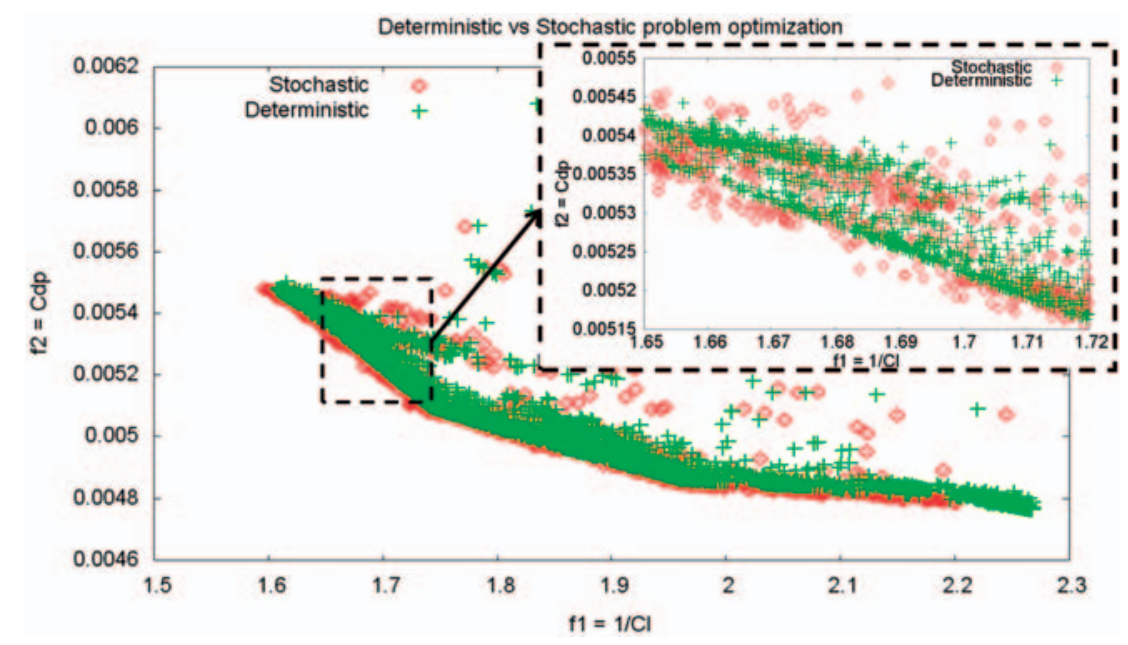

Fig. 16 Comparison between deterministic and stochastic results 


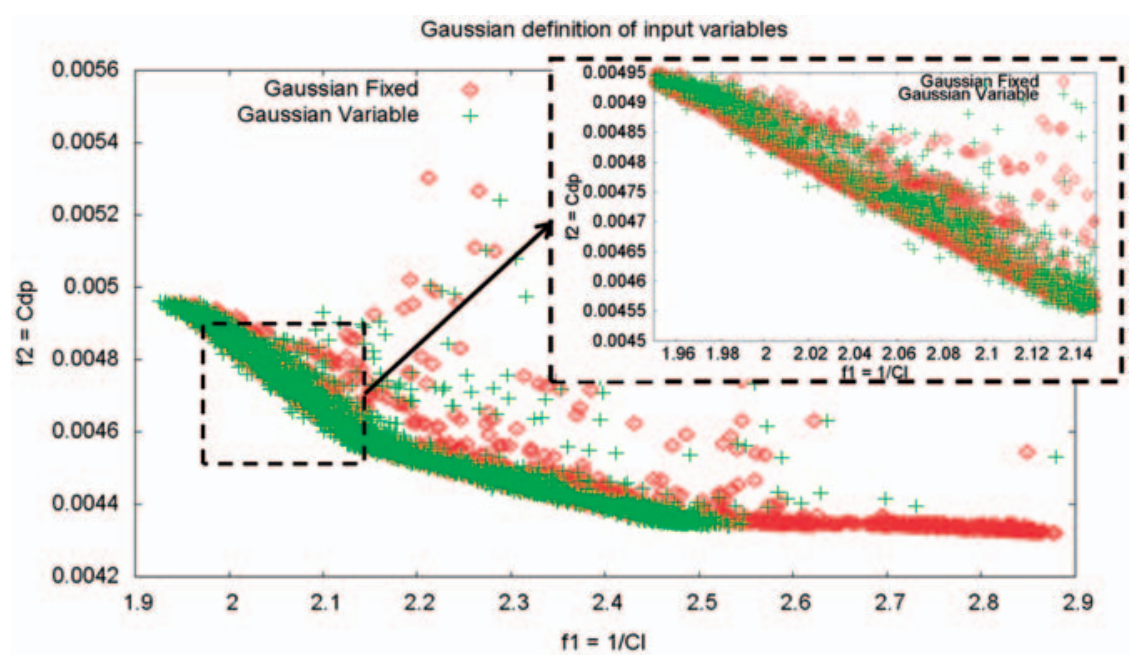

Fig. 17 Comparison between random variable and fixed definition and detail on random variable definition effects on the front

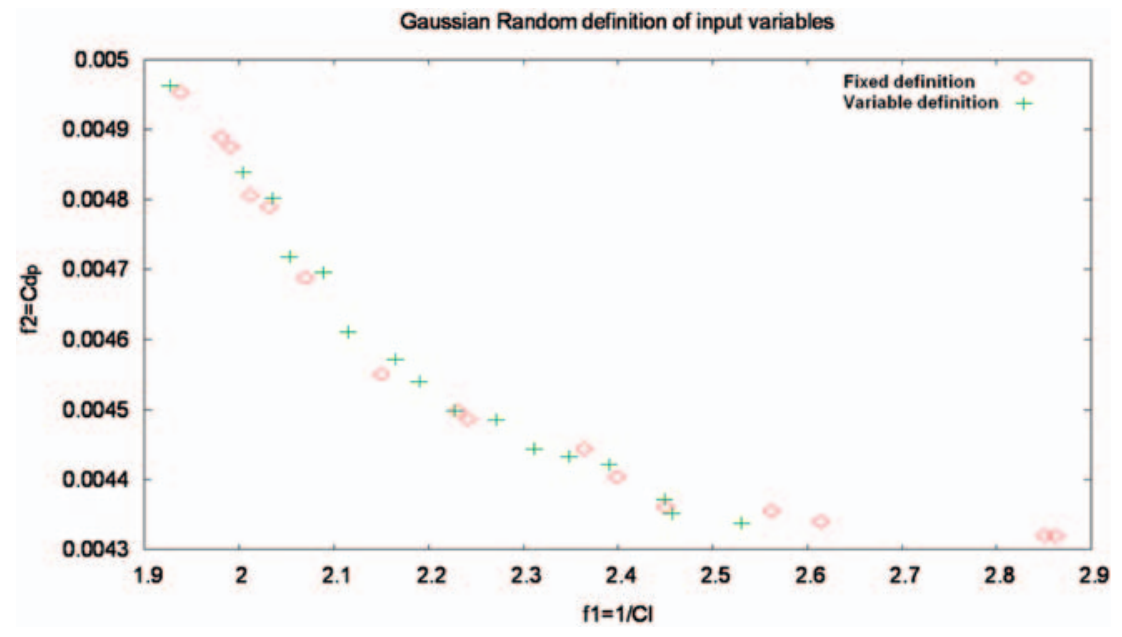

Fig. 18 Comparison between Pareto fronts for random variable and fixed definition

obtained with the variable definition. The variable definition leads to a narrower front, producing results with lower values for $f_{1}$.

A comparison of two different types of PDF has also been done. Gaussian and Uniform PDF have been used and applied to the analysis of each individual during the optimization loop and the authors have not detected any relevant difference between them.

\section{STOCHASTIC AERO-ELASTIC DESIGN OPTIMIZATION}

\subsection{Multi-objective deterministic optimization}

A deterministic aero-elastic optimization problem is now used as a basis of a multi-objective optimization. The solution of this case has been used as a reference to be compared with the stochastic optimization presented in a later subsection. The problem is based on a RAE2822 profile, which is the baseline design to solve a problem which is mainly intended to look for the smoother behaviour of the time evolution of the angular displacement $\left(\theta_{i}(t)\right)$ and the time evolution of the drag coefficient $\left(C_{\mathrm{dp}}(t)\right)$; smoother in the sense to reduce the total integral of the curvature, the second derivative of each time function. The problem can be formulated as

Minimize

$$
\begin{aligned}
& f_{1}=\sum_{i=0}^{N_{t}} \frac{\partial^{2} \theta_{i}(t)}{\partial t^{2}} \\
& f_{2}=\sum_{i=0}^{N_{t}} \frac{\partial^{2} C_{d i}(t)}{\partial t^{2}}
\end{aligned}
$$


Being $N_{t}$ the number of time steps used to calculate both time-dependant variables; so, the total sum of the curvature values is obtained and used as fitness function.

Considering the following bounds for the design variables:

(a) range of the $x$-coordinate of the elastic axis; $x$-EA: [0.25 0.65];

(b) range of the $x$-coordinate of the centre of gravity; $x$-CG: [0.35 0.60];

(c) range of the mass ratio, $\mu$ : [30.0 65.0];

(d) range of the damping coefficient for the vertical deformation, $\xi_{h}$ : $[0.150 .35]$;

(e) range of the damping coefficient for the angular spin, $\xi_{\theta}$ : $[0.150 .45]$.

Finally, angle of attack and Mach number are defined as constant values at:

(a) angle of attack: 2.79;

(b) Mach number: 0.734 .

The optimization method will look for a shape of $\theta_{i}(t)$ and $C_{\mathrm{dp}}(t)$ curves that should look similar to that shown in Fig. 19(b), which is smoother that the one shown in Fig. 19(a). Optimal results should reach smooth shapes for both fitness functions.
The optimization procedure has defined initial populations that present a wide variability, as shown in Figs 20(a) and 21(a). After the optimization process, the best populations that fulfil the optimization criteria are obtained. Figures 20(b) and 21(b) show a set of curves with a smoother time evolution.

However, not only the smoothness of the curves is improved, but also the dispersion is reduced. Both $\theta_{i}(t)$ and $C_{\mathrm{dp}}(t)$ initial populations present a large variability: a large dispersion between members of the population, but also a significant difference between the behaviour of each member. Some of them present an early converge in time, but others converge after a larger amount of time steps. On the other hand, the behaviour of the best populations is much homogeneous, as it can be seen in Figs 20 (b) and 21(b).

The integration of the aero-elastic problem with a multi-objective optimizer provides a whole population and a Pareto front that follow the usual behaviour of this kind of analysis. Initial population has a poor behaviour, but it continuously improves on each iteration up to the optimal one as Fig. 22 shows.

The analysed deterministic optimization has been performed as a validation of the problem definition. It defines a comparison point with the next analyses to be done. Comparing the obtained results for the best
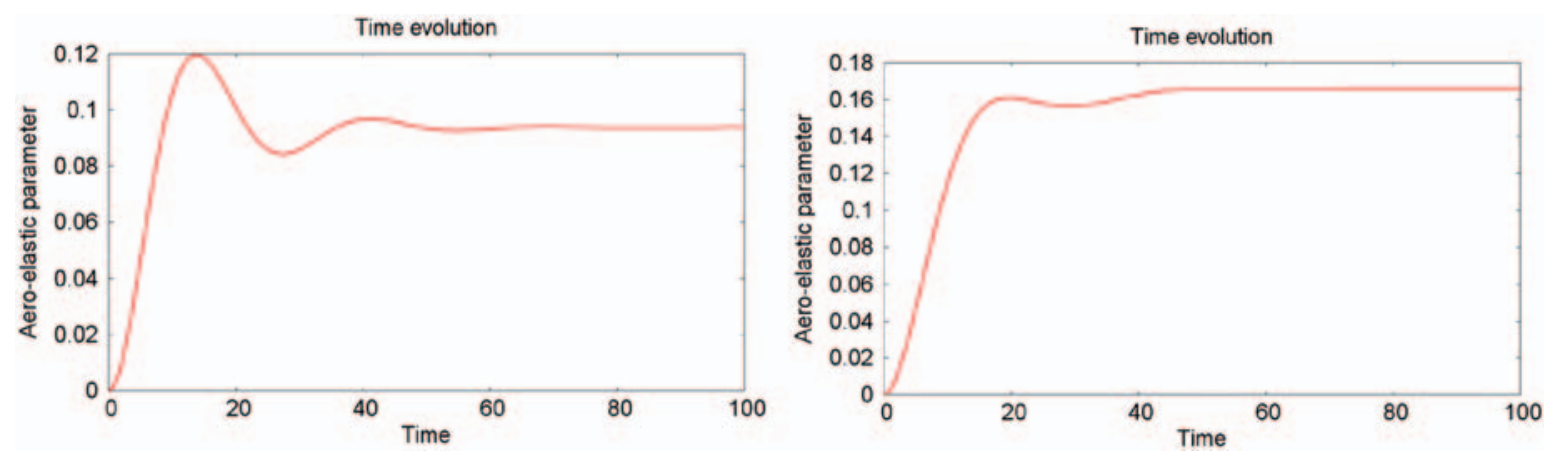

Fig. 19 Examples of (a) non-smooth and (b) smoother curves
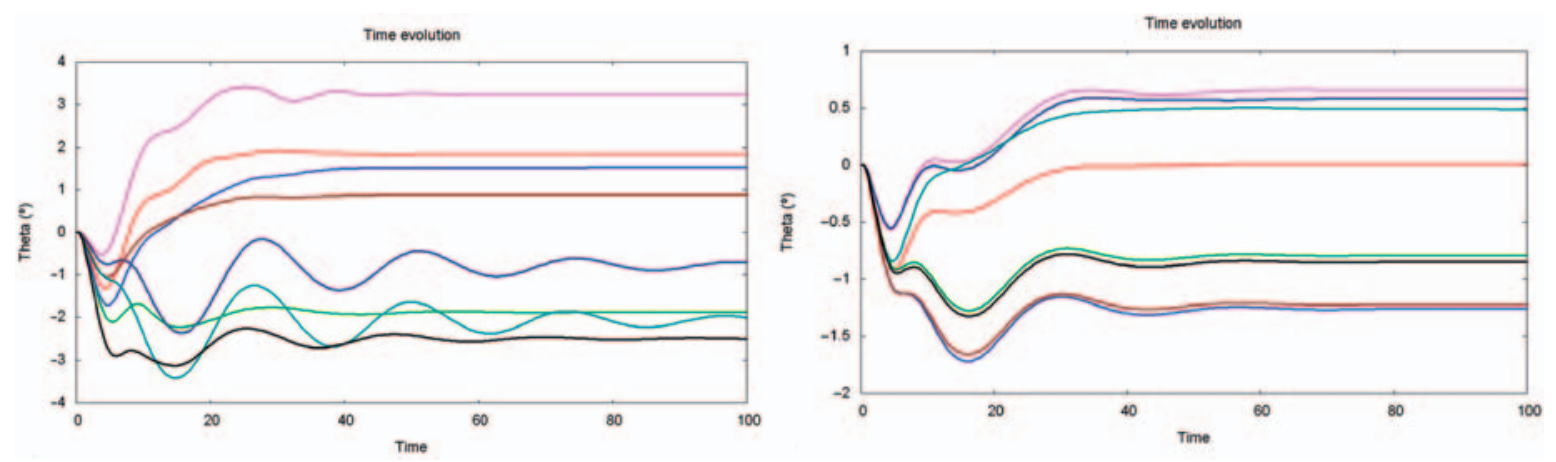

Fig. 20 (a) Initial population for $\theta_{i}(t)$ and (b) best population for $\theta_{i}(t)$ 

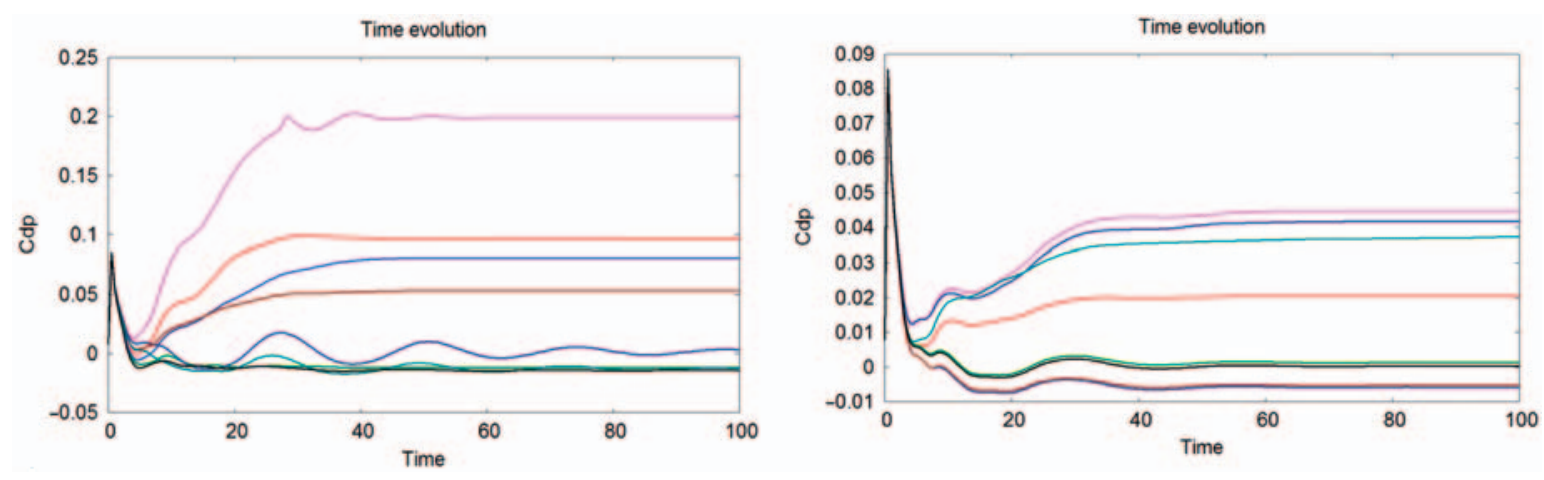

Fig. 21 (a) Initial population for $C_{\mathrm{dp}}(t)$ and (b) best population for $C_{\mathrm{dp}}(t)$
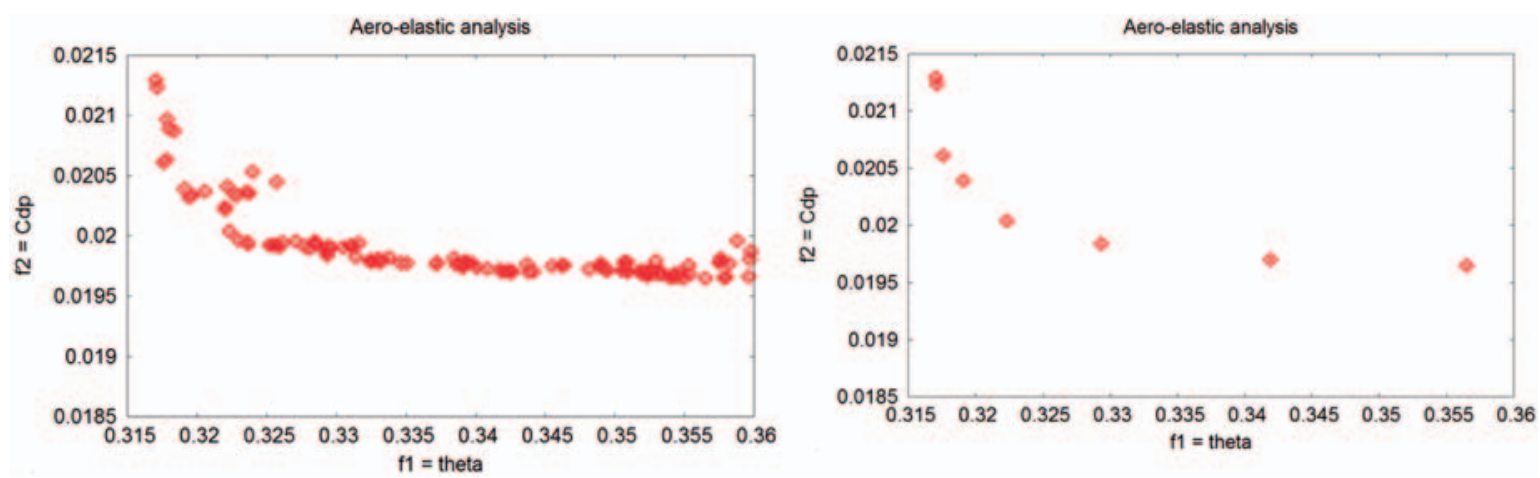

Fig. 22 Detail on (a) the whole population and (b) Pareto front from the optimization process

and the initial populations, one can realize the closer behaviour of all the optimal members compared with the initial ones. Even the dispersion is reduced; each of the optimum members has his own shape.

\subsection{Multi-objective stochastic optimization}

In this case, both objective functions are the mean values of the total sum of the curvature of two different outputs: the time evolution of the angular spin of the aero-elastic airfoil $\left(\theta_{i}(t)\right)$ and the time evolution of the drag coefficient $\left(C_{\mathrm{dp}}(t)\right)$. The analysis is aimed to compare if the stochastic procedure is able to find robust solutions without defining SD of the main output as one of its objective functions, which would be the case in a classical robust design problem.

The problem is now defined as

Minimize

$$
\begin{aligned}
& f_{1}=\operatorname{mean}\left(\sum_{i=0}^{N_{t}} \frac{\partial^{2} \theta_{i}(t)}{\partial t^{2}}\right) \\
& f_{2}=\operatorname{mean}\left(\sum_{i=0}^{N_{t}} \frac{\partial^{2} C_{d p}(t)}{\partial t^{2}}\right)
\end{aligned}
$$

$N_{t}$ being the number of time steps used to calculate angular movement; so, the total sum of the curvature values is obtained and used as fitness function.

Considering the design variables as defined in the previous deterministic aero-elastic case. Finally, angle of attack and Mach number are defined as stochastic variables.

1. Angle of attack follows a Gaussian distribution; mean: 2.79, SD: 0.01 .

2. Mach number follows a Gaussian distribution; mean: 0.734, SD: 0.01 .

Again, the problem is mainly intended to look for the smoother behaviour of both curves. Results can be analysed using a similar scheme as it has been done in the two previous cases. In Fig. 23, the detail showing the whole population identifies the main fitness function as the one with bigger dispersion. The range of obtained values is larger in $f_{1}$ case than in $f_{2}$ case. When the attention is focussed only on the Pareto front, it can be realized that both value ranges have similar dispersion.

Another comparison can be established between the time evolution of each initial individual, showing its five stochastic evaluations, and the same time evolution for the optimal individuals. Figures 24 and 25 show the time evolution of $\theta_{i}(t)$ and $C_{\mathrm{dp}}(t)$. In this 


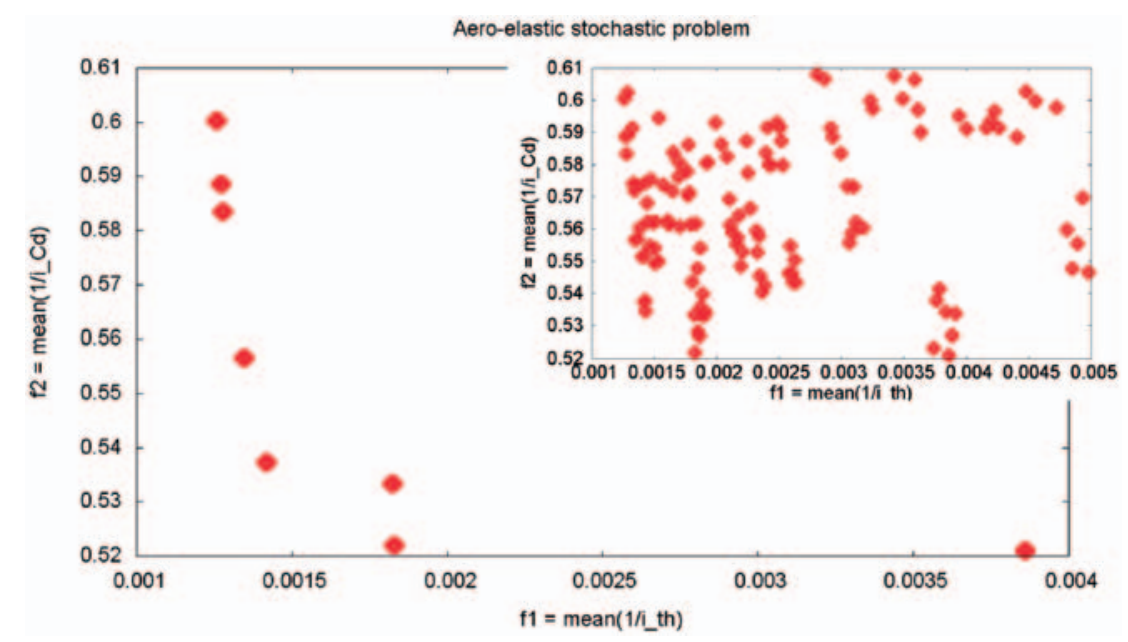

Fig. 23 Whole population and Pareto front for an aero-elastic stochastic analysis
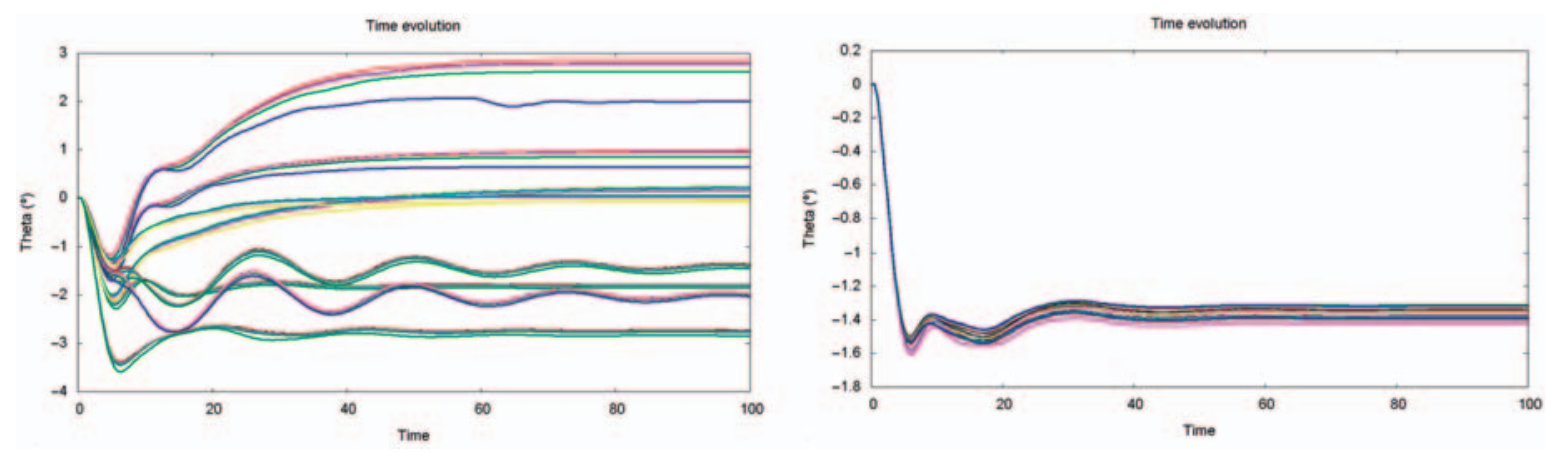

Fig. 24 (a) Initial and (b) best populations of theta, $\theta$, evolution
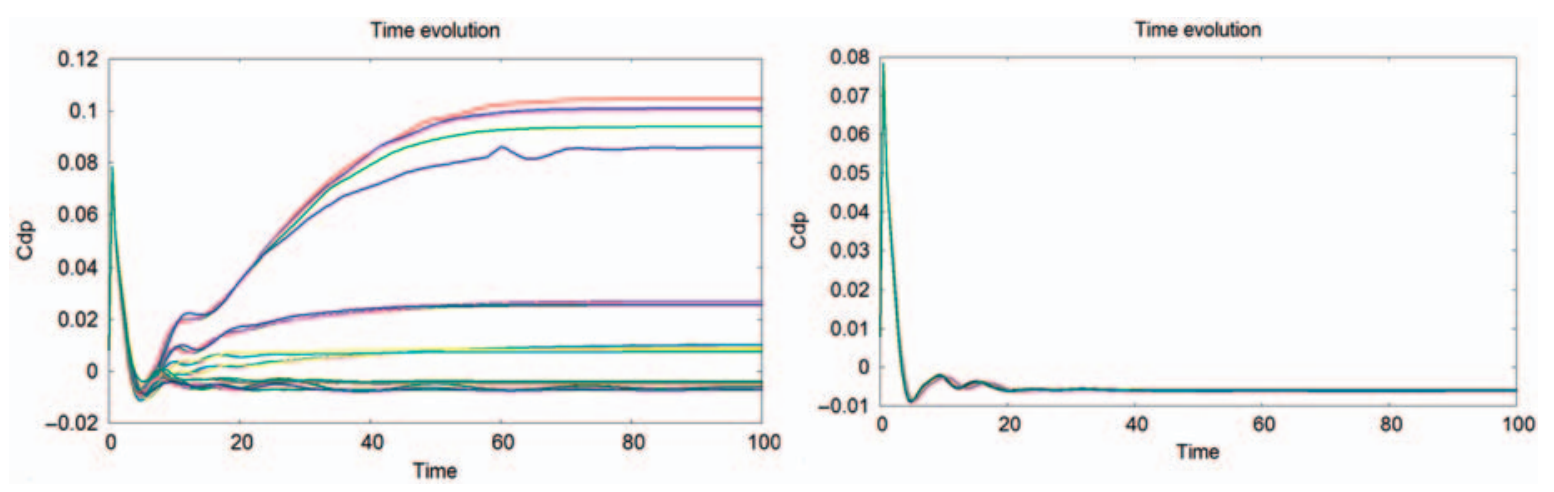

Fig. 25 (a) Initial and (b) best populations of $C_{\mathrm{dp}}(t)$ evolution

case, even not defining the SD as one of the objective functions, the final aim is reached. Both $\theta_{i}(t)$ and $C_{\mathrm{dp}}(t)$ curves became smoother after the optimization.

Similar conclusions can be taken from Figs 24 and 25 , as it has been done in the previous test case.
Comparing initial and best populations, one can realize how the optimization process tends to look for the fittest individuals. Compared with the deterministic results, the dispersion between optimal members is reduced and the shapes of all the optimal members tend to be pretty similar. 


\subsection{Multi-objective stochastic robust design optimization}

Same aero-elastic problem is now used as the basis of the stochastic optimization, based on the same problem definition already used in previous section.

The problem is now defined as

Minimize

$$
\begin{aligned}
& f_{1}=\operatorname{mean}\left(\sum_{i=0}^{N_{t}} \frac{\partial^{2} \theta_{i}(t)}{\partial t^{2}}\right) \\
& f_{2}=\sigma\left(\sum_{i=0}^{N_{t}} \frac{\partial^{2} \theta_{i}(t)}{\partial t^{2}}\right)
\end{aligned}
$$

Considering the design variables and bounds as defined in the stochastic case.

The problem is mainly intended to look for the smoother and robust behaviour of $\theta_{i}(t)$. Not only the curvature of each single individual is considered, but also the behaviour of all the stochastic set of generated individuals. As it is shown in Fig. 26, significantly different behaviours could be obtained with slightly different values of the input values.

The main difference with the deterministic optimization problem, regarding the definition of the objective functions, is the fact that now mean and SD are the selected functions. Regarding the problem definition, it is clear that the stochastic definition of the angle and Mach number are the main issue.

Results can be analysed using a similar scheme as it has been done in the deterministic case. First of all, a comparison between the initial population and the optimal one has been done. The plots in Fig. 27, showing the whole population, and Fig. 28, showing the physical meaning of these individuals, demonstrate that the initial individuals are far from the optimal values. Again, the optimization process is able to tend to the optimum.

The coupling between the stochastic procedure and the aero-elastic analysis tool does work as expected, without any problem. In Fig. 27, both the obtained Pareto front and the whole population are shown.
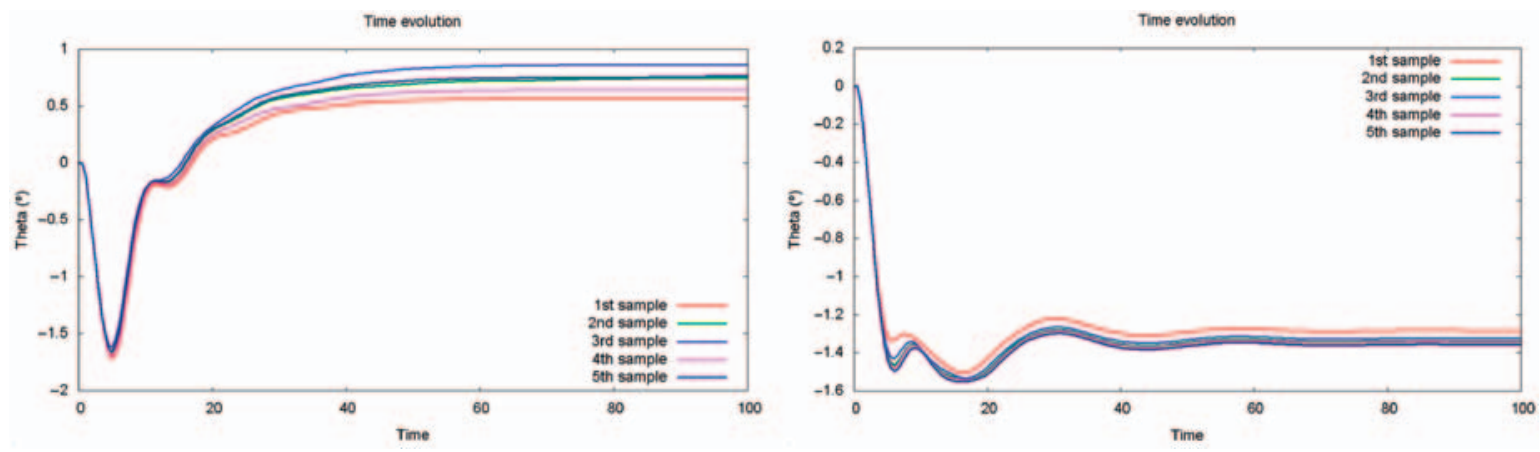

Fig. 26 ((a) and (b)) Examples of the angular movement for a set of stochastic samples

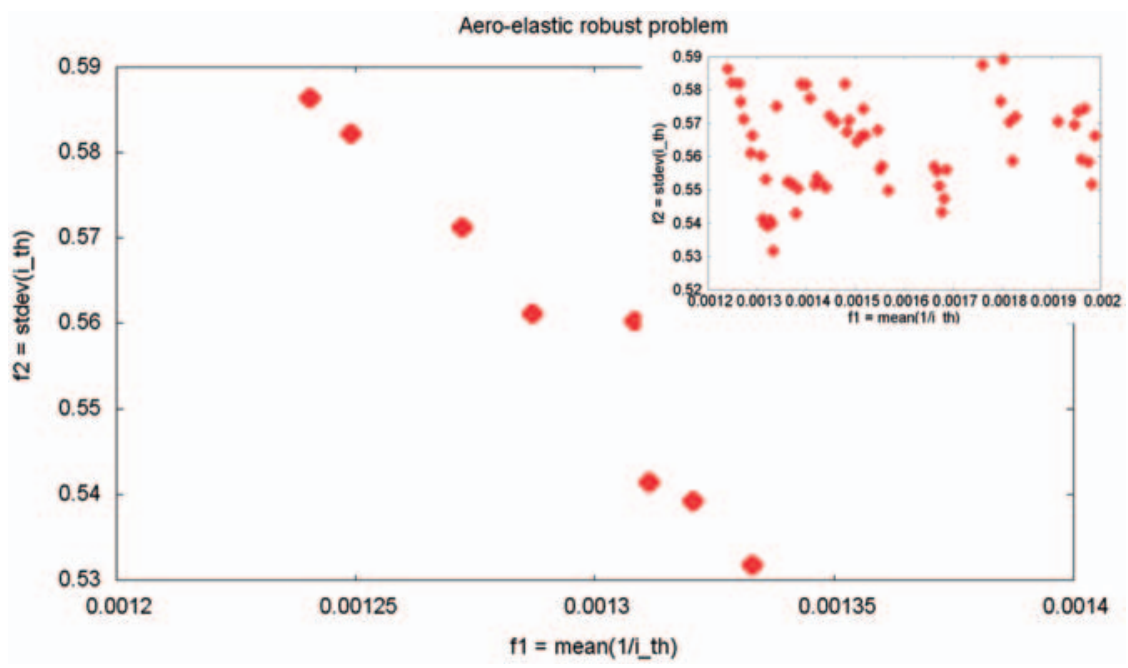

Fig. 27 Whole population and Pareto front for an aero-elastic robust analysis 

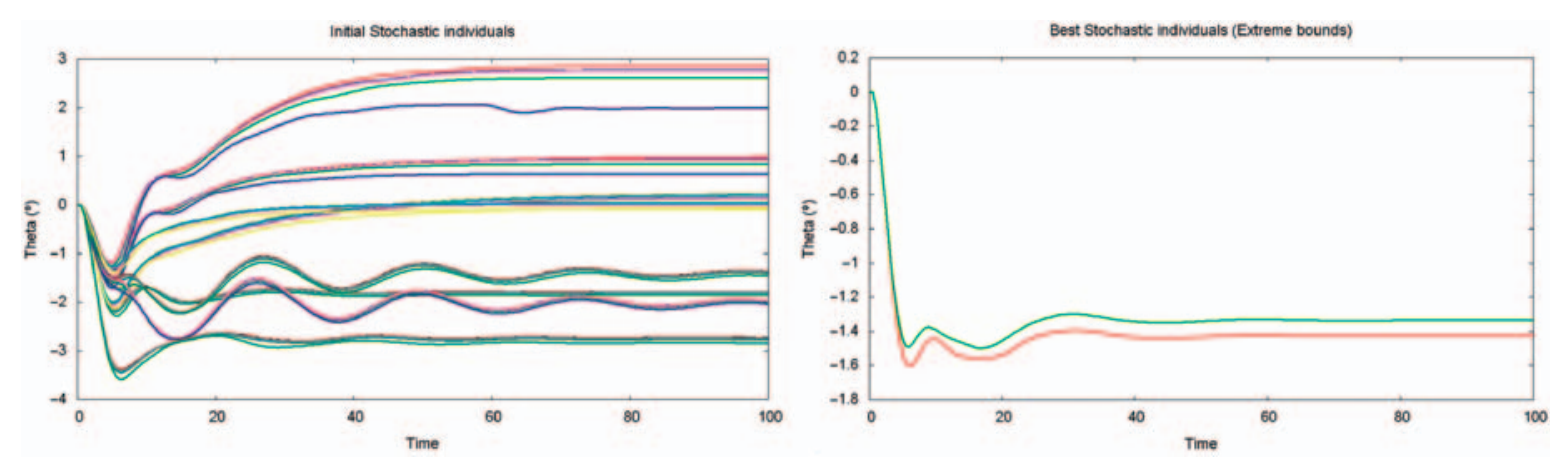

Fig. 28 (a) Initial and (b) best populations

Another comparison can be established between the time evolution of each initial individual, showing its five stochastic evaluations, and the same time evolution for the optimal individuals. It is easy to realize how the initial population is more disperse, in all the senses. Each individual differs a lot from the other ones, but also each stochastic evaluation of individuals also presents a bigger variability. On the other hand, the set of best solutions tends to the same shape, with lower variability comparing both individuals and stochastic evaluations.

Additional information taken from the plot in Fig. 28(a) and (b) of the time evolutions of theta $\theta_{i}(t)$ is of great interest to validate the final results of this test case.

Plots in Fig. 28 showing the $\theta_{i}(t)$ fitness function shapes clearly identify how the optimization process behaves. Figure 28(a) shows the initial individuals and how they do not follow any trend. Figure 28(b), where only the extreme bounds of the whole set of obtained curves are shown to simplify, demonstrates how the optimization procedure leads to soft shapes (decrease the curvature as defined), and standardizes the optimized shape to the whole set of optimal values. In this particular case, where the fitness function is strongly related to a time-dependant function, it is also important to confirm that the results adjust to the desired behaviour, as shown in Fig. 28.

Comparing the results with the deterministic or stochastic cases, the robust case increases the dispersion of the optimal values because of the change on the objective functions from the deterministic values to the mean values, and finally the mean and the SD.

A perfect coupling between aero-elastic problem and stochastic procedure has been performed. The test case does not use any geometrical information; it only uses a fixed geometry of a RAE2822 profile. The evolutionary algorithm controls other kinds of input parameters like mass ratio or damping coefficient, which are directly related with this type of problem.
An additional contribution from this test case is the use of mean and SD as fitness functions. The robustness of the solution has been reinforced by these two facts; namely the stochastic definition of the input variables, which introduces uncertainty concept into the analysis, but also using the variability as an objective of the optimization, which ensures the minimization of this variability.

\section{CONCLUSIONS}

A new stochastic optimization procedure has been defined and analysed. The integration of an evolutionary algorithm and a stochastic analysis tool has been compared with the first issues of the methodology $[19,20]$. Several options had been evaluated related to the stochastic definition of the parameters and how the uncertainty could be spread across the numerical analysis.

Three cases have been defined; the classical deterministic solution, which does not define any uncertainty at all, the stochastic one, which defines the mean values of the functional as fitness function of the optimization, and the robust one, which defines both the mean and the SD of the functional as the two fitness function to deal with during the optimization. The comparison between the deterministic, the stochastic, and the robust design cases shows how the solution deals with the uncertainty of the inputs and how a robust solution is obtained. Comparing the deterministic solutions with the stochastic and robust ones, one can realize how the introduction of the stochastic definition leads to completely new results. The stochastic and robust cases are able to reduce the dispersion of the results, while ensuring the robustness of the solution.

Monte-Carlo techniques, as well as Latin Hypercube, are computationally expensive. The use of a surrogate model, like ANN, is mandatory. If it is well trained and validated, it has been demonstrated that the approximation error of the surrogate model 
remains low enough. The intrinsic variability of the stochastic method, due to its statistical definition of the variables, is added to the error of the surrogate model. It is important, then, to keep both under control and below a desirable limit in order not to lead to wrong results. The amount of random samples defined for the stochastic definition of the input values is of big importance. A big amount of samples produces a bigger variability and a better representation of the random nature of input variables. Nevertheless, the higher the amount of samples is, the higher the computational cost is.

In order to obtain a competitive method, further work in parallelization is urgently required. In addition, new developments on collocation methods are under analysis to check if the reduction of evaluations compensates the multi-point character of these methods.

The stochastic definition has demonstrated its robustness in front of the two main cases regarding the type of objective functions, namely the stochastic case and the robust case. The first one uses the mean, while the second one uses both the mean and the SD values. Both cases have led to optimal solutions that fulfil the requirements. Due to the fact that the uncertainty propagation method is completely coupled with the evolutionary algorithm, the definition of the SD as a fitness function does not require a great effort. Robustness is ensured by the input stochastic definition, and SD provides additional information.

\section{FUNDING}

This study has been supported by the EC Commission under FP6 project NODESIM-CFD (contract number 030959) and by the Spanish Ministerio de Ciencia e Innovación through project DPI2008-05250.

\section{(C) Authors 2011}

\section{REFERENCES}

1 Helton, J. C. and Davis, F. J. Latin Hypercube sampling and the propagation of uncertainty in analyses of complex systems. Reliabil. Eng. Syst. Safety, 2003, 81(1), 23-69.

2 Durga Rao, K., Kushwaha, H. S., Verma, A. K., and Srividya, A. Quantification of epistemic and aleatory uncertainties in level-1 probabilistic safety assessment studies. Reliabil. Eng. Syst. Safety, 2007, 92(7), 947-956.

3 Hurtado, J. E. and Barbat, A. H. Monte-Carlo techniques in computational stochastics mechanics. Ed. Springer, Arch. Comput. Methods Eng., 1998, 5(1), 3-29.
4 Cook, P. H., McDonald, M. A., and Firmin, M. C. P. Aerofoil RAE 2822 -pressure distributions, and boundary layer and wake measurements, experimental data base for computer program assessment, AGARD Report AR 138, 1979.

5 Flores, R. and Ortega, E. PUMI: an explicit 3D unstructured finite element solver for Euler equations. CIMNE, 2007.

6 Drela, M. XFOIL - an analysis and design system for low Reynolds number airfoils, low Reynolds number aerodynamics. Proceedings of the Conference, Notre Dame, Indiana, Germany, 5-7 June, 1989, 1-12.

7 GiD. The pre and post-processor tool. CIMNE, available from www.gid.cimne.com (accessed April 2011).

8 Ortega, E., Oñate, E., and Idelshon, S. An improved finite point method for a three dimensional potential flows. Comput. Mech., 2007, 40, 949-963.

9 Ortega, E., Oñate, E., and Idelshon, S. A finite point method for adaptive three-dimensional compressible flow calculations. Int. J. Numer. Methods Fluids, 2009, 60, 937-971.

10 Tang, Z., Periaux, J., Bugeda, G., and Oñate, E. Lift maximization with uncertainties for the optimization of high-lift devices. Int. J. Numer. Methods Fluids, Early View, 2010, 64, 119-135.

11 Tang, Z., Periaux, J., Bugeda, G., and Oñate, E. Lift maximization with uncertainties for the optimization of high lift devices using multi-criterion evolutionary algorithms, CEC2009. 2009 IEEE Congress on Evolutionary Computation, Tronheim (Norway), 18-21 June 2009.

12 López, R. Flood A1. An open source neural networks C++ library, User Guide, CIMNE, 2007, [Department RMEE (Resistencia de Materials i Estructures a l'Enginyeria), University UPC (Universitat Politècnica de Catalunya)].

13 López, R. Neural networks for variational problems in engineering. PhD Thesis, Department RMEE (Resistencia de Materials i Estructures a l'Enginyeria), University UPC (Universitat Politècnica de Catalunya), 2008.

14 López, R. and Oñate, E. A variational formulation for the multilayer perceptron, Artificial Neural Networks, ICANN 2006. Lecture Notes Comput. Sci., 2006, 4132(I), 159-168.

15 López, R., Balsa-Canto, E., and Oñate, E. Neural networks for variational problems in engineering. Int. J. Numer. Methods Eng, Early View, 2008, 75(11), 1341-1360.

16 Deb, K., Pratap, A., Agarwal, S., and Meyarivan, T. A fast and elitist multi-objective genetic algorithm: NSGA-II. IEEE Trans. Evolut. Comput., 2002, 6(2), 182-197.

17 Papadrakakis, M., Lagaros, N. D., and Tsompanakis, Y. Structural optimization using evolution strategies and neural networks. Comput. Methods Applic. Mech. Eng., 1998, 156, 309-333.

18 Quagliarella, D. and Vicini, A. Designing high-lift airfoils using genetic algorithms. Proceedings of EUROGEN, Jyvaskyla, Finland, 30 May-3 June 1999. 
19 Pons-Prats, J., Oñate, E., Zárate, F., Bugeda, G., and Hurtado, J. Non-deterministic shape optimization in aeronautics. In Proceedings of MAO AIAA-ISSMO Conference, Victoria, Canada, AIAA, 1012 September 2009.

20 Pons-Prats, J., Bugeda, G., Oñate, E., Zárate, F., and Hurtado, J. Robust shape optimization in aeronautics. In Proceedings of WCSMO-8 Conference, Lisbon, Portugal, 2009, 1-5 June 2008.

\section{APPENDIX}

\section{Notation}

AoA angle of attack

$C_{\mathrm{dp}} \quad$ airfoil pressure drag coefficient

$C_{l} \quad$ airfoil lift coefficient $f_{1} \quad$ objective function 1

$f_{2} \quad$ objective function 2

$M \quad$ Mach number

$N_{t} \quad$ number of time steps

$\theta \quad$ theta angle; angular deformation during flutter phenomena

$\sigma \quad$ SD 